\title{
EMBEDDINGS AND IMMERSIONS OF MANIFOLDS IN EUCLIDEAN SPACE
}

\author{
BY
}

DAVID R. BAUSUM( $\left.{ }^{1}\right)$

\begin{abstract}
The problem of computing the number of embeddings or immersions of a manifold in Euclidean space is treated from a different point of view than is usually taken. Also, a theorem dealing with the existence of an embedding of $M^{m}$ in $R^{2 m-2}$ is given.
\end{abstract}

Introduction. This paper studies the existence and classification of differential immersions and embeddings of a differentiable manifold into Euclidean space from a different point of view than is frequently taken. Most authors use Hirsch's results [11] and study a Postnikov factorization of the map $B O(k) \rightarrow B O$ and try to lift the stable normal map of the manifold $M^{m} \nu: M \rightarrow B O$ back to $B O(k)$ for various $k$. See Gitler's article [4] for references of this method.

There is another technique for obtaining embeddings and immersions which is due to Haefliger and Hirsch and is described in \$2. It involves studying a Postnikov factorization of the map $P^{n} \rightarrow P^{\infty}$ and (for embeddings) trying to lift a map g: $M \times M-\Delta / Z_{2}=M^{*} \rightarrow P^{\infty}$ back to $P^{n}$. See [3], [6], [23], and [24] for authors who have used this approach. One difficulty with this method is in calculating the cohomology of $M^{*}$ in terms of something known, namely the cohomology of $M$. Now, Haefliger proved in [7] that the inclusion map $j: M^{*} \rightarrow S^{\infty} \times z_{2} M \times M$ induces an epimorphism for $Z_{2}$-cohomology. In $\$ 7$ I prove that if $m$ is odd $j^{*}$ induces isomorphisms of the $E_{r}$-terms of the Bockstein spectral sequences of the two spaces for $r \geqslant 2$. $\$ 6$ contains a calculation of the Bockstein spectral sequence of $S^{\infty} \times_{Z_{2}} X \times X$ for a finite C. W. complex $X$. Thus a good hold on $H^{*}\left(M^{*}, Z\right)$ is obtained (when $m$ is odd). Similar results are obtained for the twisted integer cohomology of $M^{*}$ when $m$ is even.

$\S 3$ describes a modified Postnikov factorization of the map $p: P^{n} \rightarrow P^{\infty}$ obtained by killing the first four nonzero homotopy groups of the fiber of $p$. It also describes the set of isotopy classes of differentiable embeddings of $M^{m}$ in

Received by the editors September 25, 1974.

AMS (MOS) subject classifications (1970). Primary 57D40; Secondary 55G45.

Key words and phrases. Embeddings and immersions, twisted cohomology operations, Postnikov-towers, Bockstein spectral sequence.

( $\left.{ }^{1}\right)$ The content of this paper is a portion of the author's Ph. D. thesis written under Professor W. S. Massey at Yale University in 1974. During the preparation of the paper the author was supported in part by a National Science Foundation Graduate Fellowship, GP-38590. 
$R^{2 m-i}\left(\operatorname{Emb}\left(M, R^{2 m-i}\right)\right)$ for $i \leqslant 2$ and the set of regular homotopy classes of immersion of $M^{m}$ in $R^{2 m-i}\left(\operatorname{Imm}\left(M, R^{2 m-i}\right)\right)$ for $i \leqslant 2$ in terms of the cohomology of $M^{*}$ and a space $U^{*}$.

$\S 4$ reviews known results on the $Z_{2}$-cohomology of $M^{*}$, and $\S \S 5$ and 8 describe the action of certain cohomology operations on $H^{*}\left(M^{*}\right)$.

$\S 9$ contains a description of $\operatorname{Imm}\left(M, R^{2 m-i}\right)(i \leqslant 2)$ for certain manifolds $M$ and a description of $\operatorname{Emb}\left(M, R^{2 m}\right)$ in terms of the cohomology of $M$. The embeddings and immersions of real projective space $P^{m}$ in $R^{2 m-i}(i \leqslant 2)$ are also determined.

The last section contains a theorem on the existence of an embedding of $M^{m}$ in $R^{2 m-2}$. For example, it shows that if $\bar{w}_{m-i}=0$ for $i \leqslant 4$ and either $m \equiv 0,1$ (4) and $M$ is orientable or $m \equiv 2,3$ (4) and $M$ is nonorientable then there always exists such an embedding.

Note that $M$ is always a compact, connected, differentiable manifold of dimension $m$.

1. The purpose of this section is to establish notation that will be used in the next few sections. Let $T$ be the category of topological spaces and continuous functions. If $B$ is a fixed space, there is a category $T_{B}$ which consists of "spaces over $B$ ". The objects of $T_{B}$ are maps in $T, X \rightarrow B$, and a morphism from $X \rightarrow B$, to $Y \rightarrow B$ is just a commutative triangle

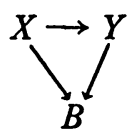

in $T$. A homotopy in $T_{B}$ is a family of maps $g_{t}: X \rightarrow Y$ which makes the obvious triangle commute for each $t$. If $g: X \rightarrow B$ and $Y$ is an object in $T_{B},[X, Y ; g]$ denotes the set of homotopy classes of maps in $T_{B}$ from $X$ to $Y$.

A second category is $T(B)$ which consists of "spaces over and under $B$ ". An object of $T(B)$ consists of two maps, $s: B \rightarrow X$ and $p: X \rightarrow B$ with $p s=1_{B}$. The morphisms and the notion of homotopy in $T(B)$ are the obvious ones. If $X$ and $Y$ are two objects in $T(B),\langle X, Y\rangle$ denotes the set of homotopy classes of maps in $T(B)$ from $X$ to $Y$. The important thing is that many of the techniques of homotopy theory extend to the category $T(B)$. The results and definitions needed in this paper are summarized below; the reader should refer to $[12, \S 2]$ or [17, Part 2] for the proofs of these results or for additional properties of the category $T(B)$.

First there is the notion of fibration in $T(B)$. To an object $X$ in $T(B)$ there is associated a "path fibration" $\Omega_{B} X \rightarrow P_{B} X \rightarrow X . \Omega_{B} X$ and $P_{B} X$ are spaces in $T(B)$ (i.e. they come with maps $s$ and $p$ ), and the maps $\Omega_{B} X \rightarrow P_{B} X$ and $P_{B} X \rightarrow X$ are both maps in $T(B)$. The definition of $P_{B} X$ is given for the sake of 
completeness: $P_{B} X=\{f: I \rightarrow X \mid f(0) \in s(B)$ and $p f(t)=p f(0) \forall t \in I\}$; recall $s: B \rightarrow X$ and $p: X \rightarrow B$. The reader should note two things about $P_{B} X$. First, $P_{B} X$ need not be contractible. Second, if $F$ is the fiber (in $T$ ) of the map $p$ : $X \rightarrow B$, then $\Omega F$ is the fiber (in $T$ ) of the map $P_{B} X \rightarrow X$. An additional property of this construction is that if $f: X \rightarrow Y$ is a map in $T(B)$, then there is a fibration in $T(B), E_{B} f \rightarrow X \rightarrow Y$. Looping (in $T(B)$ ) the last sequence produces a long sequence of fibrations in $T(B)$, and if the function $\left\langle Z,{ }_{-}\right\rangle(Z$ is an object in $T(B)$ ) is applied to it, a long exact sequence of groups and sets is obtained.

A final property of this path space construction that is needed is the following. If $X$ is a space (in $T$ ) then $B \times X$ becomes an object in $T(B)$ by letting $B \times X \rightarrow B$ be the projection map and $B \rightarrow B \times X$ be given by choosing a base point in $X$. In this case $P_{B}(B \times X)=B \times P X$ and $\Omega_{B}(B \times X)=B \times \Omega X(P X$ and $\Omega X$ are the usual path and loop spaces of $X$ ).

If $X$ is an object in $T_{B}$ (or $T(B)$ ), $H^{*}\left(X ; Z_{2}\right)$ is a module not only over the mod 2 Steenrod algebra $A$, but also over the ring $A(B)=H^{*}\left(B ; Z_{2}\right) \odot A$ (see [16] for a discussion of $\odot$, the semitensor product). An element $y \otimes a \in A(B)$ acts on $x \in H^{*}\left(X: Z_{2}\right)$ by $y \otimes a \cdot x=g^{*}(y) \cup a(x)$ (recall $g: X \rightarrow B$ ) and is called a twisted primary cohomology operation. Relations in $A(B)$ give rise to twisted secondary operations; they are studied in [17].

What follows is a brief description of cohomology with local coefficients; see $[18, \S 3]$ or [15] for more details. Suppose $f: \Gamma \rightarrow \operatorname{Aut}(H)$ is a homomorphism of a group $\Gamma$ into the automorphism group of an abelian group $H$. From the principal $\Gamma$-bundle $\Gamma \rightarrow E(\Gamma, 1) \rightarrow K(\Gamma, 1)$, construct the space $K(H, n ; f)=$ $E(\Gamma, 1) \times_{f} K(H, n)$. There is a fibration $K(H, n) \stackrel{j}{\longrightarrow} K(H, n ; f) \stackrel{\pi}{\longrightarrow} K(\Gamma, 1)$, which has a natural section $s$, and if $g: X \rightarrow K(\Gamma, 1)$ is a map, the set $[X, K(H, n ; f) ; g]$ turns out to be isomorphic to the $n$-dimensional local cohomology of $X$ with coefficients $\left\{H_{x}\right\}$ determined by $\pi_{1}(X) \rightarrow \Gamma \rightarrow \operatorname{Aut}(H)$ as defined by Steenrod. This cohomology group is written $H^{n}\left(X ; \mathrm{Hg}^{*}(f)\right)$. The "fundamental class" of $K(H, n ; f)$ is constructed in [18, p. 4]. It is the (unique) class $e^{\prime}$ in $H^{n}\left(K(H, n ; f) ; H \pi^{*}(f)\right)$ with $j^{*}\left(e^{\prime}\right)$ the fundamental class in $H^{n}(K(H, n) ; H)$ and $s^{*}\left(e^{\prime}\right)=0(s: K(\Gamma, 1) \rightarrow K(H, n ; f))$. Note that if $f$ acts trivially on $H$, then $K(H, n ; f)=K(\Gamma, 1) \times K(H, n)$ and $[X, K(H, n ; f) ; g] \simeq$ $[X, K(H, n)] \simeq H^{n}(X ; H)$, the usual cohomology group of $X$, as it should be. The case of interest here is $\Gamma=Z_{2}, H=Z$, and $f: Z_{2} \rightarrow \operatorname{Aut}(Z)$ an isomorphism, in which case $H^{n}\left(X ; Z g^{*}(f)\right)$ will be abbreviated to $H^{n}(X ; Z g)\left(g: X \rightarrow K\left(Z_{2}, 1\right)\right)$. In this case the twisted (or local) fundamental class $e^{\prime} \in H^{n}(K(Z, n ; f) ; Z \pi)$ $\left(\pi: K(Z, n ; f) \rightarrow K\left(Z_{2}, 1\right)\right)$ is a class of infinite order which restricts to the fundamental class of $H^{n}(K(Z, n) ; Z)$.

2. If $M$ is a manifold, let $\Delta=\{(x, x) \mid x \in M\}$ be the diagonal, and set $M^{*}=M \times M-\Delta / Z_{2}$. There is a $Z_{2}$-covering $Z_{2} \rightarrow M \times M-\Delta \rightarrow M^{*}$ which 
has a classifying map $g: M^{*} \rightarrow P^{\infty}$. The following theorem is a consequence of work of Haefliger [8].

TheOREM 1. Let $2 n>3(m+1)$.

(a) There exists an embedding of $M^{m}$ in $R^{n}$ if and only if there exists a lift of $g: M^{*} \rightarrow P^{\infty}$ to $P^{n-1}$.

(b) If there is one embedding of $M$ in $R^{n}$, then $\operatorname{Emb}\left(M, R^{n}\right) \cong\left[M^{*}, P^{n-1} ; g\right]$.

A similar result is true for immersions. First, let $U \subset M \times M$ be a $Z_{2}$ equivariant tubular neighborhood of the diagonal $\Delta$. Set $U^{*}=U-\Delta / Z_{2}$. Note that $U^{*} \subset M^{*}$ and that the classifying map of the $Z_{2}$-covering $Z_{2} \rightarrow U-\Delta \rightarrow U^{*}$ is just the composite $U^{*} \rightarrow M^{*} \rightarrow P^{\infty}$. That composite is also called $g$. The following theorem is a consequence of work of Haefliger and Hirsch [9].

TheOREM 2. Let $2 n>3(m+1)$.

(a) There exists an immersion of $M$ in $R^{n}$ if and only if there exists a lift of $g: U^{*} \rightarrow P^{\infty}$ to $P^{n-1}$.

(b) If there is one immersion of $M$ in $R^{n}$, then $\operatorname{Imm}\left(M, R^{n}\right) \cong\left[U^{*}, P^{n-1} ; g\right]$.

It should be mentioned that $U^{*}$ has the same homotopy type as the total space of the projective bundle with fiber $P^{m-1}$ associated to the tangent bundle of $M$.

3. The first step in applying the two theorems of the previous section is to compute the first few terms in a modified Postnikov factorization of the map $p: P^{n} \rightarrow P^{\infty}$. Thomas [21] describes the procedure if the map $p$ is simple, and McClendon [18] extends the construction to the nonsimple case, i.e. when there is a nontrivial action of $\pi_{1}$ of the base on the fiber. Since the fiber of $p$ is the $n$-sphere, and the action of $\pi_{1}\left(P^{\infty}\right)=Z_{2}$ on $S^{n}$ is the antipodal map, $p$ will be simple if $n$ is odd, and nonsimple if $n$ is even. In fact in the $n$ even case the only place (in the range of the factorization considered) that the nonsimple action plays a role is at the first stage. That step is considered in some detail, and the results in the other cases will be given without proof.

So let $n$ be even, $n \geqslant 8$, and $f: Z_{2} \rightarrow \operatorname{Aut}(Z)$ be an isomorphism. Then the first space $E_{1}$ in the factorization of $P^{n} \rightarrow P^{\infty}$ is obtained by "killing" the transgression of a generator $\left(\alpha_{n}\right)$ of $H^{n}\left(S^{n} ; Z\right)$. One would like to say that $\tau\left(\alpha_{n}\right) \in H^{n+1}\left(P^{\infty} ; Z g\right)$, use it to pull back the path fibration over $K(Z, n+1 ; f)$, and thus obtain $E_{1} \rightarrow P^{\infty}$. The problem is there are two fibrations over $K(Z, n+1 ; f)$ which could be pulled back. The relationship between them needs to be emphasized. The first, $K(Z, n ; f) \rightarrow \bar{P} K(Z, n+1 ; f) \stackrel{\pi}{\longrightarrow} K(Z, n+1 ; f)$ consists of maps and spaces over $P^{\infty}$ and is the path fibration in the category $T\left(P^{\infty}\right)$ over $K(Z, n+1 ; f)\left(\bar{P}=P_{P^{\infty}}\right.$ and $\left.\Omega_{P^{\infty}} K(Z, n+1 ; f)=K(Z, n ; f)\right)$. The second, *: $K(Z, n) \rightarrow \bar{P} K(Z, n+1 ; f) \stackrel{\pi}{\longrightarrow} K(Z, n+1 ; f)$, is obtained by making 
$\pi$ into a fibration in the category $T$ and looking at the fiber. In fact the second classifies fibrations (in $T$ ) with fiber $K(Z, n)$ which have a nontrivial action of $\pi_{1}$ of the base on the fiber (see [19, Theorem 2.2]). Thus it should be used to "kill" $\tau\left(\alpha_{n}\right)$. The question then becomes what is the total space $\left(E^{\prime}\right)$ over $P^{n}$ obtained by pulling * back by the composite $P^{n} \stackrel{p}{\longrightarrow} P^{\infty} \stackrel{\tau\left(\alpha_{n}\right)}{\longrightarrow} K(Z, n+1 ; f)$ ? Note that $\tau\left(\alpha_{n}\right) p$ is not null homotopic (it induces an isomorphism of fundamental groups); however, it does represent an element in $H^{n+1}\left(P^{n} ; Z p\right)$ (which is the zero group). Thus $\tau\left(\alpha_{n}\right) p$ is homotopic (in $T_{P^{\infty}}$ ) to the "constant map" $s p$ (s: $P^{\infty} \rightarrow K(Z, n+1 ; f)$ is the canonical section), and so $E^{\prime}$ has the same homotopy type as the space $E^{\prime \prime}$ obtained by pulling * back by $s p$. But an inspection of the definition of $E^{\prime \prime}$ shows that it is just $P^{n} \times_{P^{\infty}} K(Z, n ; f)$ (the fiber product of $P^{n} \rightarrow P^{\infty}$ with $\left.K(Z, n ; f) \rightarrow P^{\infty}\right)$. Thus the following diagrams (whose columns are fibrations in $T$ ) are obtained.

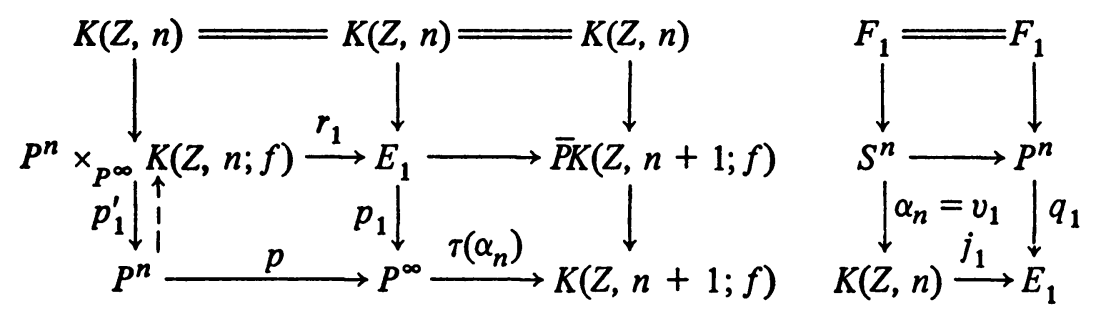

Since the first few homotopy groups of $F_{1}$ are 2-primary, the $Z_{2}$-cohomology of $E_{1}$ must be computed through a certain range. Now both the fibrations $p_{1}$ and $p_{1}^{\prime}$ are simple for $Z_{2}$-cohomology in dimensions $<2 n+1$, so Thomas' paper [21] is applicable. In particular it is easy to see that the hypotheses of his Property 5 (pp. 15 and 16) are satisfied, so there is an exact sequence $\left(Z_{2}\right.$-coefficients everywhere until further notice) for $n<t \leqslant 2 n$ :

$$
0 \rightarrow H^{t}\left(E_{1}\right) \stackrel{r_{1}^{*}}{\longrightarrow} H^{t}\left(P^{n} \times_{P^{\infty}} K(Z, n ; f)\right) \stackrel{\tau}{\longrightarrow} H^{t+1}\left(P^{\infty}\right) .
$$

One way to get the $Z_{2}$-cohomology of $P^{n} \times_{p^{\infty}} K(Z, n ; f)$ is to compute the $Z_{2}$-cohomology of $K(Z, n ; f)$ and then study the natural map $P^{n} \times{ }_{P^{\infty}}$ $K(Z, n ; f) \rightarrow P^{\infty} \times_{P^{\infty}} K(Z, n ; f)$. The Serre spectral sequence of the fibration $K(Z, n) \rightarrow K(Z, n ; f) \rightarrow P^{\infty}$ (with $Z_{2}$-coefficients) has

$$
E_{2}^{p, q}=H^{p}\left(P^{\infty} ; H^{q}\left(K(Z, n) ; Z_{2}\right) 1^{*}(\phi)\right)
$$

where $\phi$ is the $\pi_{1}\left(P^{\infty}\right)$ action on $H^{*}\left(K(Z, n) ; Z_{2}\right)$. But that action is trivial since it fixes the $Z_{2}$ fundamental class $i_{n}\left(H^{n}\left(K(Z, n) ; Z_{2}\right)=Z_{2}\right)$ and thus must fix $\mathrm{Sq}^{I} i_{n}$ and all products. Also, $i_{n}=j^{*}\left(e_{n}\right)\left(e_{n} \in H^{n}\left(K(Z, n ; f) ; Z_{2}\right)\right.$ is the reduction of $e^{\prime}$ from $\left.\S 1\right)$ so $\tau\left(i_{n}\right)=0$. Thus the above spectral sequence collapses and $E_{2}=E_{\infty}$. This observation is the main fact needed to write down the following basis (valid for any $n \geqslant 7)$ of $H^{i}\left(K(Z, n ; f), Z_{2}\right)$ in dimensions $i \leqslant n+6$ (note 
that $a^{i}=\pi^{*}\left(u^{i}\right)$ where $\pi: K(Z, n ; f) \rightarrow P^{\infty}$, and $u \in H^{1}\left(P^{\infty}, Z_{2}\right)$ is a generator, and that $e_{n}$ is abbreviated to just $e$ ):

$$
\begin{array}{cll}
\text { dimension } & & \multicolumn{1}{c}{\text { relations }} \\
\leqslant n-1 & a^{i} & \\
n & e, a^{n} & \\
n+1 & a e, a^{n+1} & \mathrm{Sq}^{1} e=a e \\
n+2 & \mathrm{Sq}^{2} e, a^{2} e, a^{n+2} & \\
n+3 & \mathrm{Sq}^{3} e, a \mathrm{Sq}^{2} e, a^{3} e, a^{n+3} & \mathrm{Sq}^{2,1} e=a \mathrm{Sq}^{2} e+a^{3} \\
n+4 & \mathrm{Sq}^{4} e, a \mathrm{Sq}^{3} e, a^{2} \mathrm{Sq}^{2} e, & \mathrm{Sq}^{3,1} e=a^{2} \mathrm{Sq}^{2} e+a \mathrm{Sq}^{3} e \\
& a^{4} e, a^{n+4} & \\
n+5 & \mathrm{Sq}^{5} e, a \mathrm{Sq}^{4} e, a^{2} \mathrm{Sq}^{3} e, & \mathrm{Sq}^{4,1} e=a \mathrm{Sq}^{4} e+a^{2} \mathrm{Sq}^{3} e \\
& a^{3} \mathrm{Sq}^{2} e, a^{5} e, a^{n+5} & \\
n+6 & \mathrm{Sq}^{6} e, \mathrm{Sq}^{4,2} e, a \mathrm{Sq}^{5} e, & \mathrm{Sq}^{5,1} e=a \mathrm{Sq}^{5} e+a^{2} \mathrm{Sq}^{4} e \\
& a^{2} \mathrm{Sq}^{4} e, a^{3} \mathrm{Sq}^{3} e, a^{6} e, & \\
& a^{4} \mathrm{Sq}^{2} e, a^{n+6} &
\end{array}
$$

The other fact needed to get this basis is that if $\mathrm{Sq}_{a}^{1}$ is the composite of the boundary (in cohomology) of the coefficient sequence $0 \rightarrow Z_{f} \rightarrow Z_{f} \rightarrow Z_{2}$ $\rightarrow 0$ with reduction mod 2 , then $0=\mathrm{Sq}_{a}^{1}(e)=\mathrm{Sq}^{1} e+a e$. The first equality follows because $e$ is the reduction of the class $e^{\prime} \in H^{n}\left(K(Z, n ; f) ; Z \pi^{*} f\right)$; the last equality is a consequence of [5] and gives the first relation in the table. It easily follows that $H^{*}\left(P^{n} \times_{P^{\infty}} K(Z, n ; f)\right) \simeq H^{*}(K(Z, n ; f)) /\left\{a^{k} \mid k>n\right\}$ in dimensions $\leqslant n+6$.

Thomas shows that $\tau$ is a map of $A\left(P^{\infty}\right)$ modules, so $\tau\left(\pi^{*}\left(u^{i}\right) \mathrm{Sq}^{j} e\right)=$ $u^{i} \mathrm{Sq}^{j} \tau(e)$. Since $\tau(e)=u^{n+1}$, it is easy to compute $\tau$ on the above set of elements and thus obtain $H^{i}\left(E_{1}\right)=\operatorname{ker} \tau$ if $i \leqslant n+6$.

At this point it is straightforward to "kill" the next three nonzero homotopy groups of $S^{n}$. The $Z_{8}$ which occurs in dimension $n+3$ can be killed in three steps by using the method described by Thomas in [21]. Thus if $X$ is a C. W. complex and $g: X \rightarrow P^{\infty}$, the resulting factorization can in fact be used to study the existence of a lift of $g$ to $P^{n}$ provided $\operatorname{dim} X \leqslant n+5$ (this uses $\pi_{n+4}\left(S^{n}\right)=$ $\pi_{n+5}\left(S^{n}\right)=0$ ). The following diagram illustrates the factorization that is obtained. The $k$-invariants $\left(g_{i} j_{i}, i=1,2,3\right)$ may be read off the table which occurs later in this section and so are not included here.

The second step in applying the two theorems of $\S 2$ is to change Diagram 1 , which is a factorization in the category $T$ of the map $p: P^{n} \rightarrow P^{\infty}$, into 
Diagram 2, which is a factorization in the category $T\left(P^{n}\right)$ of the map $\bar{p}:\left(P^{n}\right)^{2}$ $\rightarrow P^{n}\left(\left(P^{n}\right)^{2}\right.$ is obtained by pulling $p$ back along $\left.p\right)$.

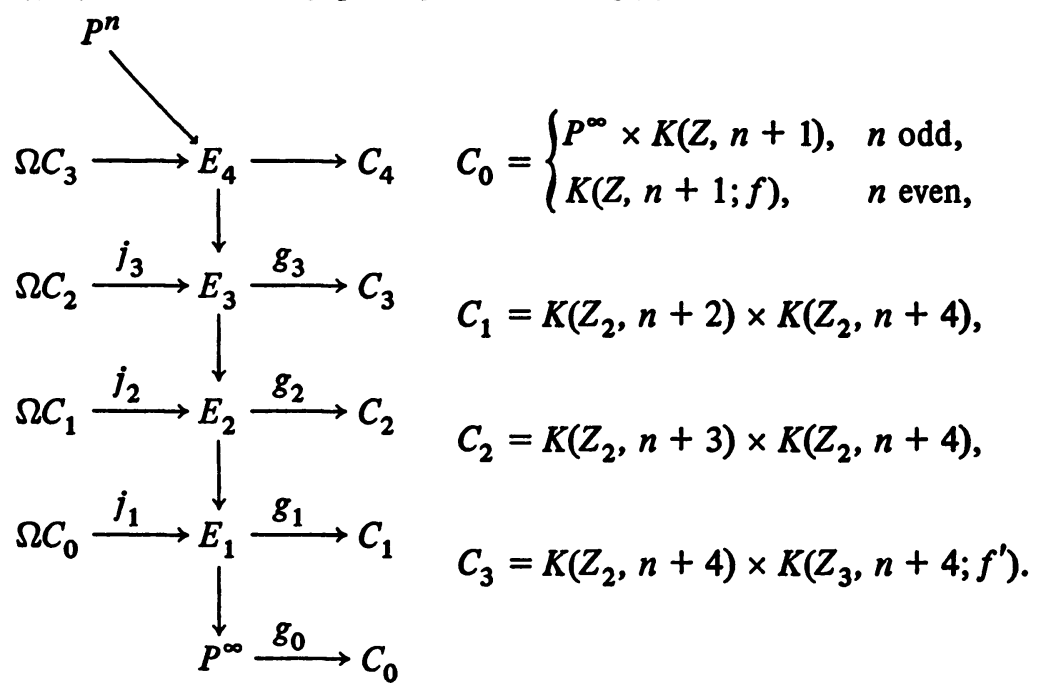

Diagram 1

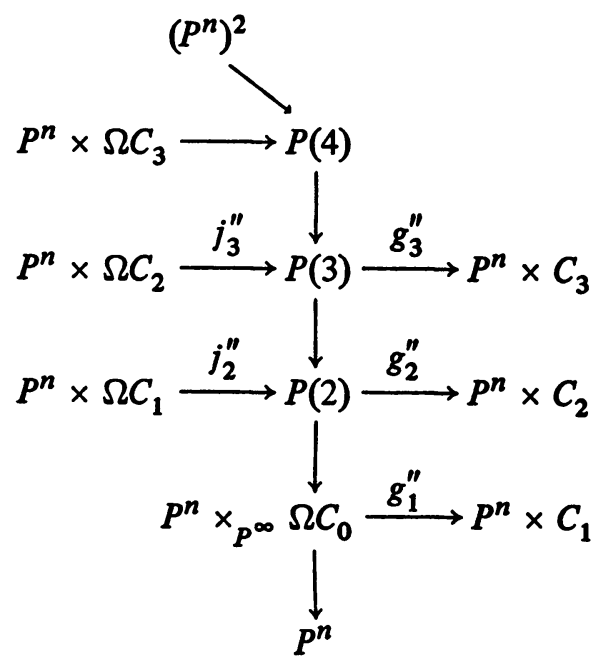

\section{DiAGRAM 2}

Note that the spaces $C_{i}$ in Diagram 2 are the same as the ones in Diagram 1 and that

$$
\Omega C_{0}= \begin{cases}P^{\infty} \times K(Z, n), & n \text { odd, } \\ K(Z, n ; f), & n \text { even. }\end{cases}
$$

This is a formal procedure, the details of which may be found in [17] in case $p$ is simple and in [18] in the nonsimple case. The idea behind this transformation is the following. 
Let $g: X \rightarrow P^{\infty}$, and $G: X \rightarrow P^{n}$ be a lift of $g$ so that $f=p G$. Then it is easy to see that there is a set equivalence $\left[X, P^{n} ; g\right] \cong\left[X,\left(P^{n}\right)^{2} ; G\right]$. The first set uses the map $p: P^{n} \rightarrow P^{\infty}$ while the second uses $\bar{p}:\left(P^{n}\right)^{2} \rightarrow P^{n}$. Now $\bar{p}$ has a canonical section $s$ given by $s(x)=(x, x)$ for $x \in P^{n}$, so $\left(P^{n}\right)^{2}$ is in fact an object of the category $T\left(P^{n}\right)$. Also, there is a natural way to make $X \vee P$ ( $V$ is the wedge) into an object of $T\left(P^{n}\right)$, and it is easy to check that $\left\langle X \vee P^{n},\left(P^{n}\right)^{2}\right\rangle$ $\cong\left[X,\left(P^{n}\right)^{2} ; G\right]$. Thus if the functor $\left\langle X \vee P^{n},-\right\rangle$ is applied to Diagram 2, a collection of exact sequences (which is actually part of an exact couple) is obtained and may be used to describe $\left\langle X \vee P^{n},\left(P^{n}\right)^{2}\right\rangle$ provided that $\operatorname{dim} X \leqslant n$ +5 . The one thing needed for this is the description of the maps $g_{k}^{\prime \prime} j_{k}^{n}$. That is provided by the following table which is a consequence of the factorization of $p: P^{n} \rightarrow P^{\infty}$ described above. Note that the description depends on the congruence class of $n \bmod 8$.

THEOREM 3.

$$
\begin{aligned}
& g_{1}^{\prime} \text { is }\left(1 \otimes \mathrm{Sq}^{2} i_{n}+x^{2} \otimes i_{n}, 1 \otimes \mathrm{Sq}^{4} i_{n}\right) \\
& n \equiv 1 \text { (8) } \\
& \left(1 \otimes \mathrm{Sq}^{2} i_{n}, 1 \otimes \mathrm{Sq}^{4} i_{n}+x^{4} \otimes i_{n}\right) \\
& n \equiv 3 \\
& \left(1 \otimes \mathrm{Sq}^{2} i_{n}+x^{2} \otimes i_{n}, 1 \otimes \mathrm{Sq}^{4} i_{n}+x^{2} \otimes \mathrm{Sq}^{2} i_{n}\right) \quad n \equiv 5 \\
& \left(1 \otimes \mathrm{Sq}^{2} i_{n}, 1 \otimes \mathrm{Sq}^{4} i_{n}\right) \\
& n \equiv 7 \\
& \left(1 \otimes \mathrm{Sq}^{2} i_{n}, 1 \otimes \mathrm{Sq}^{4} i_{n}\right) \\
& n \equiv 0 \\
& \left(1 \otimes \mathrm{Sq}^{2} i_{n}+x^{2} \otimes i_{n}, 1 \otimes \mathrm{Sq}^{4} i_{n}\right) \quad n \equiv 2 \\
& \left(1 \otimes \mathrm{Sq}^{2} i_{n}, 1 \otimes \mathrm{Sq}^{4} i_{n}+x^{4} \otimes i_{n}\right) \quad n \equiv 4 \\
& \left(1 \otimes \mathrm{Sq}^{2} i_{n}+x^{2} \otimes i_{n}, 1 \otimes \mathrm{Sq}^{4} i_{n}+x^{4} \otimes i_{n}\right) \quad n \equiv 6 \\
& g_{2}^{\prime} j_{2}^{\prime \prime} \text { is }\left(1 \otimes \mathrm{Sq}^{2} i_{n+1}+x^{2} \otimes i_{n+1}, 1 \otimes \mathrm{Sq}^{2,1} i_{n+1}+1 \otimes \mathrm{Sq}^{1} i_{n+3}\right) n \equiv 1 \\
& \left(1 \otimes \mathrm{Sq}^{2} i_{n+1}, 1 \otimes \mathrm{Sq}^{2,1} i_{n+1}+1 \otimes \mathrm{Sq}^{1} i_{n+3}\right) \quad n \equiv 3 \\
& \left(1 \otimes \mathrm{Sq}^{2} i_{n+1}+x^{2} \otimes i_{n+1}, 1 \otimes \mathrm{Sq}^{2,1} i_{n+1} \quad n \equiv 5\right. \\
& \left.+1 \otimes \mathrm{Sq}^{1} i_{n+3}+x^{2} \otimes \mathrm{Sq}^{1} i_{n+1}\right) \\
& \left(1 \otimes \mathrm{Sq}^{2} i_{n+1}, 1 \otimes \mathrm{Sq}^{2,1} i_{n+1}+1 \otimes \mathrm{Sq}^{1} i_{n+3}\right) \quad n \equiv 7 \\
& \left(1 \otimes \mathrm{Sq}^{2} i_{n+1}+x \otimes \mathrm{Sq}^{1} i_{n+1}+x^{2} \otimes i_{n+1}, 1 \otimes \mathrm{Sq}^{2,1} i_{n+1} \quad n \equiv 0\right. \\
& \left.+x^{2} \otimes \mathrm{Sq}^{1} i_{n+1}+1 \otimes \mathrm{Sq}^{1} i_{n+3}+x \otimes i_{n+j}\right) \\
& \left(1 \otimes \mathrm{Sq}^{2} i_{n+1}+x \otimes \mathrm{Sq}^{1} i_{n+1}, 1 \otimes \mathrm{Sq}^{2,1} i_{n+1}+1 \otimes \mathrm{Sq}^{1} i_{n+3} \quad n \equiv 2\right. \\
& \left.+x^{2} \otimes \mathrm{Sq}^{1} i_{n+1}+x^{3} \otimes i_{n+1}+x \otimes i_{n+3}\right) \\
& \left(1 \otimes \mathrm{Sq}^{2} i_{n+1}+x \otimes \mathrm{Sq}^{1} i_{n+1}+x^{2} \otimes i_{n+1}, 1 \otimes \mathrm{Sq}^{2,1} i_{n+1} \quad n \equiv 4\right. \\
& \left.+1 \otimes \mathrm{Sq}^{1} i_{n+3}+x^{2} \otimes \mathrm{Sq}^{1} i_{n+1}+x \otimes i_{n+3}\right) \\
& \left(1 \otimes \mathrm{Sq}^{2} i_{n+1}+x \otimes \mathrm{Sq}^{1} i_{n+1}, 1 \otimes \mathrm{Sq}^{2,1} i_{n+1} \quad n \equiv 6\right. \\
& \left.+x^{3} \otimes i_{n+1}+1 \otimes \mathrm{Sq}^{1} i_{n+3}+x \otimes i_{n+3}+x^{2} \otimes \mathrm{Sq}^{1} i_{n+1}\right)
\end{aligned}
$$




$$
\begin{array}{ll}
g_{3}^{\prime} j_{3}^{\prime \prime} i s\left(1 \otimes \mathrm{Sq}^{2} i_{n+2}+1 \otimes \mathrm{Sq}^{1} i_{n+3}+x^{2} \otimes i_{n+2}, 0\right) & n \equiv 1(8) \\
\left(1 \otimes \mathrm{Sq}^{2} i_{n+2}+1 \otimes \mathrm{Sq}^{1} i_{n+3}, 0\right) & n \equiv 3 \\
\left(1 \otimes \mathrm{Sq}^{2} i_{n+2}+1 \otimes \mathrm{Sq}^{1} i_{n+3}+x^{2} \otimes i_{n+2}, 0\right) & n \equiv 5 \\
\left(1 \otimes \mathrm{Sq}^{2} i_{n+2}+1 \otimes \mathrm{Sq}^{1} i_{n+3}, 0\right) & n \equiv 7 \\
\left(1 \otimes \mathrm{Sq}^{2} i_{n+2}+1 \otimes \mathrm{Sq}^{1} i_{n+3}+x \otimes i_{n+3}+x^{2} \otimes i_{n+2}, 0\right) & n \equiv 0 \\
\left(1 \otimes \mathrm{Sq}^{2} i_{n+2}+1 \otimes \mathrm{Sq}^{1} i_{n+3}+x \otimes i_{n+3}, 0\right) & n \equiv 2 \\
\left(1 \otimes \mathrm{Sq}^{2} i_{n+2}+x^{2} \otimes i_{n+2}+1 \otimes \mathrm{Sq}^{1} i_{n+3}+x \otimes i_{n+3}, 0\right) & n \equiv 4 \\
\left(1 \otimes \mathrm{Sq}^{2} i_{n+2}+1 \otimes \mathrm{Sq}^{1} i_{n+3}+x \otimes i_{n+3}, 0\right) & n \equiv 6
\end{array}
$$

The final description of the set $\left[X, P^{n} ; g\right]$ is only given when $X$ is either $M^{*}$ or $U^{*}$. These are the cases of interest in the embedding and immersion problems, and the description for them is less involved than in the general case. Actually, there are six cases of interest here; they correspond to the number of embeddings (immersions) of $M^{m}$ in $R^{2 m-i} \quad(i=0,1,2)$. First the results for embeddings are given. Then the changes needed for immersions are listed.

Proposition 4. Let $M$ be a manifold of dimension $m \geqslant 8$. Assume there is an embedding of $M$ in $R^{2 m-2}$. Then $\operatorname{Emb}\left(M, R^{2 m-2}\right)$ has an abelian group structure on it, and there exist groups $F_{1}$ and $F_{2}$ with

(1) $0 \subset F_{2} \subset F_{1} \subset F_{0}=\operatorname{Emb}\left(M, R^{2 m-2}\right)$, and

(2) $F_{2}=\operatorname{coker} \psi, F_{1} / F_{2}=\operatorname{coker} A^{2 m-4}, F_{0} / F_{1}=\operatorname{ker} A^{2 m-3}$.

The definition of the operations $A^{i}$ and $\psi$ are given in the proof.

Proof. Define $A^{i}: H^{i}\left(M^{*}, Z\right) \rightarrow H^{i+2}\left(M^{*}, Z_{2}\right)$ by

$$
A^{i}(\gamma)= \begin{cases}\mathrm{Sq}^{2} \gamma, & m \text { odd } \\ \mathrm{Sq}^{2} \gamma+g^{*}(u)^{2} \cdot \gamma, & m \text { even. }\end{cases}
$$

There is a relation $A^{2 m-2} A^{2 m-4}=0$ (note $A^{i}$ is defined on integral classes) and the operation $\psi$ is the secondary operation corresponding to that relation. Thus $\psi: \operatorname{ker} A^{2 m-4} \subset H^{2 m-4}\left(M^{*}, Z\right) \rightarrow H^{2 m-1}\left(M^{*}, Z_{2}\right)$.

The reader should now either prove the proposition by applying the functor $\left\langle M^{*} \vee P^{2 m-3},-\right\rangle$ to Diagram 2 with $n=2 m-3$ as described above or refer to [17, Theorem 3, p. 210]. In either case the fact that the cohomology of $M^{*}$ vanishes in dimensions greater than $2 m-1$ must be used.

The case of embedding $M$ in $R^{2 m-1}$ is covered by the next proposition. It may be proved the same way Proposition 4 is, and so the proof is omitted.

Proposition 5. Let $M$ be a manifold of dimension $m \geqslant 6$. Assume there exists an embedding of $M$ in $R^{2 m-1}$ (this is always the case except for certain nonorientable manifolds of dimension $\left.2^{k}\right)$. Then $\operatorname{Emb}\left(M, R^{2 m-1}\right)$ has an abelian group structure on it and there exists a group $G_{1}$ with 
(1) $0 \subset G_{1} \subset G_{0}=\operatorname{Emb}\left(M, R^{2 m-1}\right)$,

(2) $G_{1} \simeq \operatorname{coker} A^{2 m-3}$, and $G_{0} / G_{1} \simeq H^{2 m-2}\left(M^{*} ; Z g\right)$.

Note. The operation $A^{2 m-3}$ which occurs in Proposition 5 is defined as follows:

$$
\begin{array}{r}
A^{2 m-3}: H^{2 m-3}\left(M^{*} ; Z g\right) \rightarrow H^{2 m-1}\left(M^{*} ; Z_{2}\right), \\
A^{2 m-3}(\gamma)= \begin{cases}\mathrm{Sq}^{2} \gamma, & m \text { odd } \\
\mathrm{Sq}^{2} \gamma+g^{*}(u)^{2} \cdot \gamma, & m \text { even. }\end{cases}
\end{array}
$$

The case of embedding $M$ in $R^{2 m}$ is covered by

Proposition 6. Let $M$ be a manifold of dimension $m \geqslant 4$. Then $\operatorname{Emb}\left(M, R^{2 m}\right)=H^{2 m-1}\left(M^{*} ; Z\right)$.

Each of the above propositions is valid for the study of immersions once the following two changes are made. First, replace every occurrence of $M^{*}$ by $U^{*}$; second, in place of "embedding" read "immersion".

Finally, observe that the inclusion map $R^{n} \rightarrow R^{n+1}$ induces a map $\theta: \operatorname{Emb}\left(M^{m}, R^{n}\right) \rightarrow \operatorname{Emb}\left(M^{m}, R^{n+1}\right)$ and a similar map for immersions. For $n=2 m-i(i=1,2)$ the map $\theta$ preserves the filtrations defined on each set in the above propositions, and on the quotients of the filtrations is just multiplication by $g^{*}(\hat{u})$, where $\hat{u} \in H^{1}\left(P^{\infty}, Z g\right)$ is the generator of the one dimensional twisted integer cohomology of $P^{\infty}$. This fact is not exploited in the remainder of this paper and so is not proved.

4. This section contains facts and notation used in the remainder of this paper. All cohomology groups are taken with $Z_{2}$-coefficients unless other coefficients are indicated. The first theorem concerns the $Z_{2}$-cohomology of $M^{*}$ and is due to Haefliger [7]; an improvement of it is due to Yo and appeared in [26]. Both papers deal with the following commutative diagram due to Haefliger.

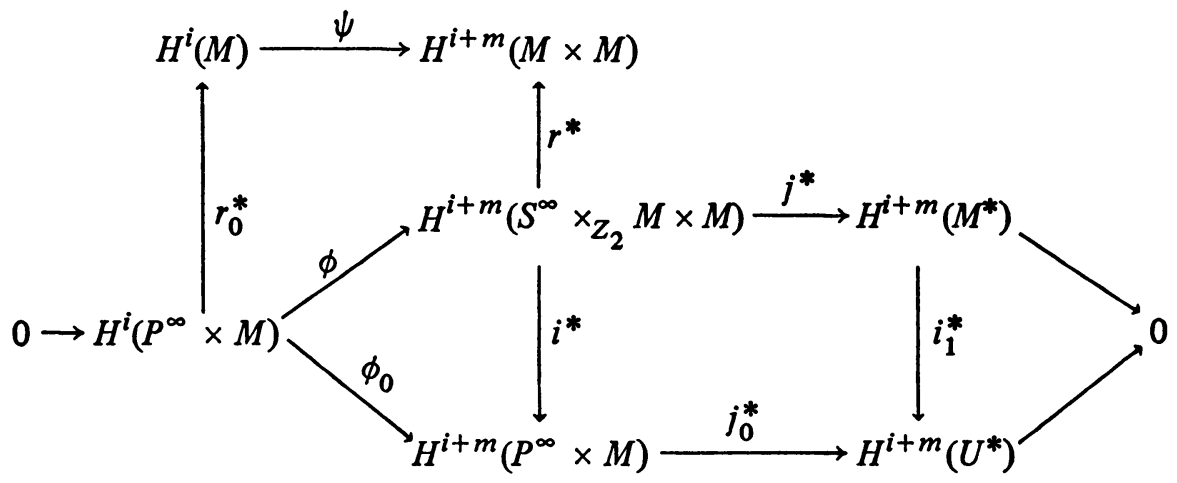

In the diagram the rows are exact, and the maps $r^{*}, r_{0}^{*}, i^{*}$, and $j^{*}$ come from the following diagram whose columns are fiber bundles. 


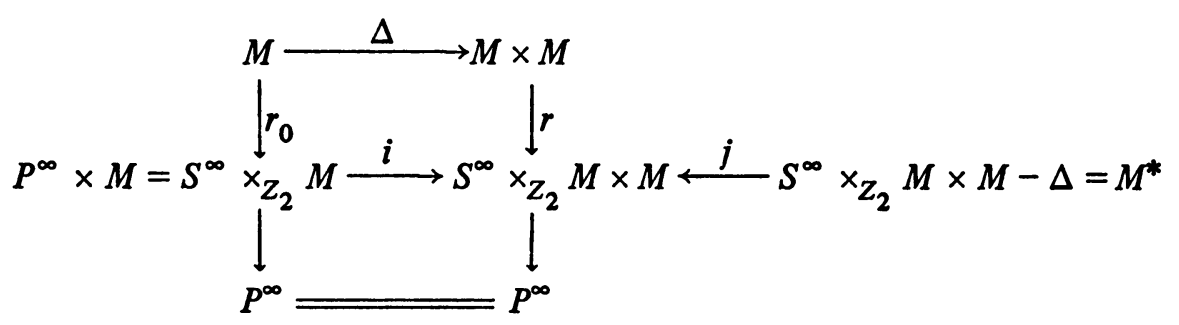

Haefliger shows an element $\alpha \in H^{*}\left(S^{\infty} \times z_{2} M \times M\right)$ is in the kernel of $j^{*}=$ image of $\phi$ if and only if there is an element $\beta \in H^{*-m}\left(P^{\infty} \times M\right)$ with $i^{*}(\alpha)=\phi_{0}(\beta)$ and $r^{*}(\alpha)=\psi r_{0}^{*}(\beta)$.

Steenrod has studied the cohomology of $S^{\infty} \times z_{2} M \times M$ and the maps $i^{*}$ and $r^{*}$. His result is the following:

(1) $H^{*}\left(S^{\infty} \times z_{2} M \times M\right) \simeq H^{*}\left(Z_{2} ; H^{*}(M \times M)\right) \simeq H^{*}\left(P^{\infty}\right) \otimes D \oplus N$, where $D=\left\{x^{2}=x \otimes x \mid x \in H^{*}(M)\right\}$ and $N=\left\{x \otimes y+y \otimes x \mid x, y \in H^{*}(M)\right\}$ are subsets of $H^{*}(M \times M)$.

(2) $i^{*}$ is a map of $H^{*}\left(P^{\infty}\right)$-modules and $\left.i^{*}\right|_{N}=0$.

(3) If $x \in H^{q}(M)$, then $i^{*}\left(1 \otimes x^{2}\right)=\Sigma_{j=0}^{q} u^{q-j} \otimes \mathrm{Sq}^{j} x$ (where $u \in H^{1}\left(P^{\infty}\right)$ is the generator).

(4) $r^{*}$ is the natural projection $H^{*}\left(Z_{2} ; H^{*}(M \times M)\right) \rightarrow H^{0}\left(Z_{2} ; H^{*}(M \times M)\right)$ $=D \oplus N$.

An easy consequence of 3 is that the map $i^{*}$ restricted to $H^{*}\left(P^{\infty}\right) \otimes D$ is a monomorphism.

The map $r_{0}^{*}$ is the obvious projection, and Haefliger gave the following description of the maps $\psi$ and $\phi_{0}$. Let $w=1+w_{1}+w_{2}+\cdots$ be the total Steifel-Whitney class of $M$ (unless stated otherwise $w_{i}$ always refers to the tangent bundle of $M)$. Then $\phi_{0}$ is a map of $H^{*}\left(P^{\infty}\right)$-modules, and if $x \in H^{q}(M)$, $\phi_{0}(1 \otimes x)=\left[u^{*} \otimes w \cdot x\right]_{q+m}=\sum_{j=0}^{m-q} u^{m-j} \otimes w_{j} \cdot x$, and $\psi(x)=(x \otimes 1) \cdot \delta_{2}$ where $\delta_{2}$ is the Poincaré-dual of $M$ in $M \times M$ (i.e. $\delta_{2}=$ P.D. $\Delta_{*}$ ([M]), where P.D.: $H_{m}(M \times M) \rightarrow H^{m}(M \times M)$ is the Poincare duality isomorphism, $[M] \in H_{m}(M)$ is a generator, and $\Delta: M \rightarrow M \times M)$. Thus each of the maps in Haefliger's theorem is described with the exception of $\phi$.

While the theorem allows the computation of $H^{*}\left(M^{*}\right)$ in theory, it is quickly seen that it is hard to obtain specific results even for spaces with nice cohomology like projective space. A more complete description of the image of $\phi$ is needed; it is provided in [26]. In order to describe Yo's result, certain operations introduced in [25] must be described.

Proposition 7 [25, p. 1481]. If $M$ is a manifold, there exist operations $Q^{i}: H^{q}(M) \rightarrow H^{q+i}(M)(i \geqslant 0)$ with the following properties:

(1) If $x \in H^{q}(M)$, then $Q^{i}(x)=0$ for $i>m-q / 2$.

(2) $Q^{0}=$ identity. 
(3) For any $z \in H_{*}(M)$ and $x \in H^{*}(M),\left\langle\mathrm{Sq}^{i} x, z\right\rangle=\left\langle x \cdot Q^{i}\right.$ P.D. $\left.z,[M]\right\rangle$ $(l$,$\rangle is the Kronecker pairing, and P.D. and [M]$ are as above.)

The $Q^{i}$ operations are given further meaning by the indentities in the next proposition. Let $\mathrm{Sq}=1+\mathrm{Sq}^{1}+\mathrm{Sq}^{2}+\cdots$ and $Q=1+Q^{1}+Q^{2}+\cdots$; then it is possible to define operators $\overline{\mathrm{Sq}}=1+\overline{\mathrm{Sq}}^{1}+\overline{\mathrm{Sq}}^{2}+\cdots$ and $\bar{Q}=1+$ $\bar{Q}^{1}+\bar{Q}^{2}+\cdots$ by the identities Sq $\overline{\mathrm{Sq}}=\overline{\mathrm{Sq}} \mathrm{Sq}=1$ and $Q \bar{Q}=\bar{Q} Q=1$. Similarly if $v=1+v_{1}+v_{2}+\cdots$ is the total Wu class (of the tangent bundle of $M)$ and $w$ the total Steifel-Whitney class, there exist classes $\bar{v}=1+\bar{v}_{1}+\bar{v}_{2}+\cdots$ and $\bar{w}=1+\bar{w}_{1}+\bar{w}_{2}+\cdots$ defined by $v \bar{v}=\bar{v} v=1$ and $w \bar{w}=\bar{w} w=1$. Recall that Wu's formula says $w=\mathrm{Sq} v$ or $v=\overline{\mathrm{Sq}} w$.

Proposition $8[25, \S 4.3]$. Let $x \in H^{*}(M)$; then

$$
\begin{gathered}
Q(x)=\overline{\mathrm{Sq}}(x) \cdot v \quad \text { or } Q^{s}(x)=\sum_{j=0}^{s} \overline{\mathrm{Sq}}^{j}(x) \cdot v_{s-j}, \\
\bar{Q}(x)=\mathrm{Sq}(x) \cdot \bar{w}, \quad \mathrm{Sq} Q(x)=x \cdot w, \quad Q \mathrm{Sq}(x)=x \cdot v, \\
\overline{\mathrm{Sq} Q}(x)=x \cdot \bar{v}, \quad \overline{Q \mathrm{Sq}}(x)=x \cdot \bar{w}, \quad Q(x \cdot y)=\overline{\mathrm{Sq}}(x) \cdot Q(y) .
\end{gathered}
$$

For example the first identity shows that the first few $Q^{i}$ are the following:

$$
\begin{aligned}
& Q^{1}(x)=\mathrm{Sq}^{1}(x)+x \cdot w_{1} \quad \text { since } v_{1}=w_{1}, \\
& Q^{2}(x)=\mathrm{Sq}^{2}(x)+w_{1} \cdot \mathrm{Sq}^{1}(x)+x \cdot \bar{w}_{2} \\
& \text { since } \mathrm{Sq}^{2}=\overline{\mathrm{Sq}}^{2} \text { and } v_{2}=w_{2}+w_{1}^{2}=\bar{w}_{2} .
\end{aligned}
$$

Yo's improvement of Haefliger's theorem can now be described. There exist maps $\xi: H^{q}\left(S^{\infty} \times z_{2} M \times M\right) \rightarrow H^{q}\left(S^{\infty} \times z_{2} M \times M\right)$ and $\bar{\xi}: H^{q}\left(P^{\infty} \times M\right)$ $\rightarrow H^{q}\left(P^{\infty} \times M\right)$ defined by

$$
\xi \mid N=1, \quad \xi\left(u^{a} \otimes x^{2}\right)= \begin{cases}\Sigma_{i=0}^{[a-1 / 2]} u^{e-2 i} \otimes\left(\bar{Q}^{i} x\right)^{2}, & a>0, \\ 1 \otimes x^{2}, & a=0,\end{cases}
$$

and

$$
\bar{\xi}\left(u^{a} \otimes x\right)=\sum_{\substack{i=0, \ldots, a \\ j=0, \ldots, a-1}} u^{a-i-j} \otimes \bar{Q}^{j} \overline{\mathrm{Sq}}^{i} x=\sum_{k=0}^{a} u^{a-k} \otimes \bar{w}_{k} \cdot x
$$

with two properties:

(1) each map is an isomorphism; in fact an inverse for $\xi$ is given by

$$
\xi^{-1} \mid N=1 \quad \text { and } \xi^{-1}\left(u^{a} \otimes x^{2}\right)= \begin{cases}\sum_{i=0}^{[a-1 / 2]} u^{a-2 i} \otimes\left(Q^{i} x\right)^{2}, & a>0, \\ 1 \otimes x^{2}, & a=0,\end{cases}
$$

and one for $\bar{\xi}$ is given by 


$$
\bar{\xi}^{-1}\left(u^{a} \otimes x\right)=\sum_{\substack{k=0, \ldots, a \\ l=0, \ldots, a-k}} u^{a-k-l} \otimes \mathrm{Sq}^{l} Q^{k} x
$$

$$
\bar{\xi} \phi_{0} H^{r}\left(P^{\infty} \times M\right)=\sum_{i=0}^{r} H^{r+m-i}\left(P^{\infty}\right) \otimes H^{i}(M), \text { and }
$$

(2) $\xi \phi H^{r}\left(P^{\infty} \times M\right)=\sum_{i=0}^{r-1} u^{r+m-2 i} \otimes d H^{i}(M)+J_{r}$, where

$u^{a} \otimes d H^{i}(M)=\left\{u^{a} \otimes x^{2} \mid x \in H^{i}(M)\right\}$ and

$$
J_{r}=\left\{u^{m-r} \otimes x^{2}+(x \otimes 1) \cdot \delta_{2} \mid x \in H^{r}(M)\right\} .
$$

Thus the maps $\bar{\xi}$ and $\xi$ induce isomorphisms

$$
H^{m+r}\left(U^{*}\right) \simeq \sum_{i=r+1}^{m} H^{m+r-i}\left(P^{\infty}\right) \otimes H^{i}(M)
$$

and

$$
H^{m+r}\left(M^{*}\right) \simeq \sum_{i=r}^{[m+r / 2]} u^{r+m-2 i} \otimes d H^{i}(M)+N^{m+r} \bmod J_{r}
$$

The second isomorphism still requires identifications to be made, but, nevertheless, is more convenient to work with than the description given by Haefliger.

5. This section contains a number of formulas which are used in the next few sections. The important ones are collected in a table at the end of this section. Note. In this section and in the rest of the paper the symbol $X^{0}$ will denote the space $S^{\infty} \times{ }_{Z_{2}} X \times X$.

Lemma 9. $Q^{2 n} Q^{1}=Q^{2 n+1}$ and $Q^{2 n+1} Q^{1}=0$.

Proof. The second fact is a consequence of the first since an easy calculation shows $Q^{1} Q^{1}=0$. The first fact is a consequence of part 3 of Proposition 7 and the Adem relation $\mathrm{Sq}^{1} \mathrm{Sq}^{2 n}=\mathrm{Sq}^{2 n+1}$.

Let

$$
L_{k}=\sum_{j=0}^{[k / 2]} \bar{Q}^{k-2 j} Q^{2 j} \text { and } D_{k}=\sum_{j=0}^{[k-1 / 2]} \bar{Q}^{k-2 j-1} Q^{2 j+1} .
$$

Note that $L_{k}+D_{k}=\Sigma_{j=0}^{k} \bar{Q}^{k-j} Q^{i}=0$ by one of Yo's results.

Lemma 10. If $k \geqslant 3$, then $L_{k}=0$ and $D_{k}=0$.

PROOF. By the remark preceding the lemma it suffices to show $L_{k}=0$. For $k=3$ that is an easy calculation. The proof for $k>3$ is by induction; so assume $0=L_{k}$. Lemma 9 and the formula $\bar{Q} Q=1$ show that 


$$
L_{k+1}=\sum_{i=0}^{k+1} \bar{Q}^{k+1-i} Q^{i}+L_{k} \cdot Q^{1}=0 .
$$

Recall that Yo's description of $H^{*}\left(M^{*}\right)$ shows that $H^{*}\left(M^{*}\right)$ contains elements of two types: first, elements $x \otimes y+y \otimes x$ and second, elements $u^{j} \otimes x^{2}$ with $\operatorname{deg} x \leqslant m-j$. If $\alpha$ stands for one of those elements, then $\mathrm{Sq}^{i} \alpha$ is gotten by considering $\alpha \in H^{*}\left(M^{0}\right)$ and looking at $\xi \mathrm{Sq}^{i} \xi^{-1}(\alpha)$. Since $\xi$ is the identity on the first set of elements, $\mathrm{Sq}^{i}$ is given by the Cartan formula on them. On the second set the definition is, in general, quite complicated. In Lemmas 12 and 13 a simple formula for $\mathrm{Sq}^{1}\left(u^{i} \otimes x^{2}\right)$ is obtained. First, $\mathrm{Sq}^{1}$ on $H^{*}\left(M^{0}\right)$ needs to be calculated.

LEMma 11. Let $x \in H^{r}(M)$ and consider $u^{i} \otimes x^{2} \in H^{*}\left(M^{0}\right)$. Then

$$
\mathrm{Sq}^{1}\left(u^{i} \otimes x^{2}\right)= \begin{cases}(i+r) u^{i+1} \otimes x^{2}, & i>0, \\ r u \otimes x^{2}+\mathrm{Sq}^{1} x \otimes x+x \otimes \mathrm{Sq}^{1} x, & i=0 .\end{cases}
$$

Proof. This is contained in Yo's paper [26]; a different proof appears later in $\S 6$.

LEMMA 12. Let $x \in H^{r}(M)$ and let $2 a+1 \leqslant m-r ;$ consider $u^{2 a+1} \otimes$ $x^{2} \in H^{*}\left(M^{*}\right)$. Then

$$
\mathrm{Sq}^{1}\left(u^{2 a+1} \otimes x^{2}\right)= \begin{cases}(r+1) u^{2 a+2} \otimes x^{2}+u^{2 a} \otimes\left(Q^{1} x\right)^{2}, & a>0 \\ (r+1) u^{2} \otimes x^{2}, & a=0\end{cases}
$$

Proof. Lemma 11 and the remark preceding it show that

$$
\begin{aligned}
\operatorname{Sq}^{1}\left(u^{2 a+1} \otimes x^{2}\right) & =\xi \operatorname{Sq}^{1} \xi^{-1}\left(u^{2 a+1} \otimes x^{2}\right) \\
& =\xi \operatorname{Sq}^{1} \sum_{i=0}^{a} u^{2 a+1-2 i} \otimes\left(Q^{i} x\right)^{2} \\
& =\xi \sum_{i=0}^{a}(r+1-i) u^{2 a+2-2 i} \otimes\left(Q^{i} x\right)^{2} \\
& =\sum_{i=0}^{a} \sum_{j=0}^{a-i}(r+1-i) u^{2 a+2-2 i-2 j} \otimes\left(\bar{Q}^{i} Q^{i} x\right)^{2}=\sum_{l=0}^{a} C_{l},
\end{aligned}
$$

where $C_{l}$ is the sum of all terms with $i+j=l$. Now

$$
C_{0}=(r+1) u^{2 a+2} \otimes x^{2}
$$

and

$$
C_{1}=r u^{2 a} \otimes\left(Q^{1} x\right)^{2}+(r+1) u^{2 a} \otimes\left(\bar{Q}^{1} x\right)^{2}=u^{2 a} \otimes\left(Q^{1} x\right)^{2}
$$

(since $\left.\bar{Q}^{1}=Q^{1}\right)$. If $l>1$, 


$$
C_{l}=\sum_{k=0}^{l}(r-k+1) u^{2 a+2-2 l} \otimes\left(\bar{Q}^{l-k} Q^{k} x\right)^{2} .
$$

But

$$
\begin{aligned}
\sum_{k=0}^{l}(r-k+1) \bar{Q}^{l-k} Q^{k} & =\sum_{k=0}^{l}(r-k+1) \bar{Q}^{l-k} Q^{k}+(r+1) \cdot \sum_{k=0}^{l} \bar{Q}^{l-k} Q^{k} \\
& =\sum_{k=0}^{l} k \bar{Q}^{l-k} Q^{k} \quad\left(-k=k \text { since everything is in } Z_{2}\right) \\
& =\sum_{i=0}^{[l-1 / 2]} \bar{Q}^{l-2 i-1} Q^{2 i+1}=0 \text { by Lemma } 10 .
\end{aligned}
$$

Thus $C_{l}=0$, if $l>1$.

Lemma 13. Let $x \in H^{r}(M)$ and let $2 a \leqslant m-r$; consider $u^{2 a} \otimes x^{2}$ in $H^{*}\left(M^{*}\right)$. Then

$$
\mathrm{Sq}^{1}\left(u^{2 a} \otimes x^{2}\right)= \begin{cases}r u^{2 a+1} \otimes x+u^{2 a-1} \otimes\left(Q^{1} x\right)^{2}+(r+a) u \otimes\left(Q^{a} x\right)^{2}, & a>0, \\ r u \otimes x^{2}+\mathrm{Sq}^{1} x \otimes x+x \otimes \mathrm{Sq}^{1} x, & a=0 .\end{cases}
$$

Proof. The case $a=0$ is just Lemma 11 , since $\xi$ and $\xi^{-1}$ are the identity on $1 \otimes x^{2}$. For $a>0$ the proof is similar to the proof of Lemma 12 and is omitted.

LeMma 14. In $H^{*}(M \times M) \mathrm{Sq}^{1} \delta_{2}=w_{1} \otimes 1 \cdot \delta_{2}$.

Proof. In order to show $\mathrm{Sq}^{1} \delta_{2}-\left(w_{1} \otimes 1\right) \cdot \delta_{2}=0$, it suffices to show

$$
\left\langle a \otimes b \cdot\left(\mathrm{Sq}^{1} \delta_{2}-\left(w_{1} \otimes 1\right) \cdot \delta_{2}\right), M \times M\right\rangle=0
$$

for all $a \otimes b \in H^{m-1}(M \times M)$. Observe that $a \otimes b \cdot \mathrm{Sq}^{1} \delta_{2}=\mathrm{Sq}^{1}\left(a \otimes b \cdot \delta_{2}\right)$ $+\mathrm{Sq}^{1}(a \otimes b) \cdot \delta_{2} ;$ also $a \otimes b \cdot \delta_{2} \in H^{2 m-1}(M \times M)$. Since $Q^{1} H^{2 m-1}(M \times M)$ $=0$, and $Q^{1}\left(\_\right)=\mathrm{Sq}^{1}\left(_{-}\right)+\underline{w}_{1}\left(\_\right)\left(\underline{w}_{1}=w_{1}(M \times M)=w_{1} \otimes 1+1 \otimes w_{1}\right)$, $\mathrm{Sq}^{1}\left(a \otimes b \cdot \delta_{2}\right)=\underline{w}_{1} \cdot a \otimes b \cdot \delta_{2}$. Thus the left-hand side of (*) becomes

$$
\begin{aligned}
\left\langle\left(\underline{w}_{1}\right.\right. & \left.\left.\cdot a \otimes b+\mathrm{Sq}^{1}(a \otimes b)-w_{1} \otimes 1 \cdot a \otimes b\right) \cdot \delta_{2}, M \times M\right\rangle \\
& =\left\langle a \otimes w_{1} b+\mathrm{Sq}^{1} a \otimes b+a \otimes \mathrm{Sq}^{1} b, \Delta_{*}(M)\right\rangle \\
& =\left\langle a \cdot w_{1} b+\mathrm{Sq}^{1} a \cdot b+a \cdot \mathrm{Sq}^{1} b, M\right\rangle \\
& =\left\langle a \cdot Q^{1} b+\mathrm{Sq}^{1} a \cdot b, M\right\rangle=\left\langle Q^{1}(a b), M\right\rangle=0 .
\end{aligned}
$$

The next to last equality is a consequence of Yo's formula $Q(a b)=\overline{\mathrm{Sq}}(a) \cdot Q(b)$.

The next lemma is a consequence of a careful reading of formula 11 in [26, p. 1787]. It is used in $\S 7$.

Lemma 15. Let $x \in H^{m-k}(M)$. If $k=2 a$, then $x \otimes 1 \cdot \delta_{2}$ is a sum of elements of the form $y \otimes z+z \otimes y$ with $y \neq z$ plus the element $1 \otimes\left(Q^{a} x\right)^{2}$. (Note: It is $1 \otimes\left(Q^{a} x\right)^{2}$ that is of interest.) 
In general, products in $H^{*}\left(M^{*}\right)$ take a complicated form. If $\alpha$ and $\beta$ are two elements in $H^{*}\left(M^{*}\right)$, their product $\alpha \cdot \beta$ is $\xi\left(\xi^{-1}(\alpha) \cdot \xi^{-1}(\beta)\right)$. For certain elements a nice description of the product may be obtained. This is dealt with in

Lemma 16. Let $x \in H^{r}(M)$, and let $i \leqslant m-r$. Consider $u \otimes 1^{2}$ and $u^{i} \otimes x^{2} \in H^{*}\left(M^{*}\right)$. Then

$$
u \otimes 1^{2} \cdot u^{i} \otimes x^{2}= \begin{cases}u^{i+1} \otimes x^{2} & \text { if } i \text { is odd or } i=0, \\ u^{i+1} \otimes x^{2}+u \otimes\left(Q^{a} x\right)^{2} & \text { if } i=2 a>0 .\end{cases}
$$

Proof. (Note: $\xi^{-1}\left(u \otimes 1^{2}\right)=u \otimes 1^{2}$.) By the remark preceding the lemma,

$$
\begin{aligned}
u \otimes 1^{2} \cdot u^{i} \otimes x^{2} & =\xi\left(u \otimes 1^{2} \cdot \sum_{j=0}^{[i-1 / 2]} u^{i-2 j} \otimes\left(Q^{j} x\right)^{2}\right) \\
& =\xi\left(\sum_{j=0}^{[i-1 / 2]} u^{i+1-2 j} \otimes\left(Q^{j} x\right)^{2}\right) \\
& =\sum_{j=0}^{[i-1 / 2]} \sum_{k=0}^{[i-2 j / 2]} u^{i+1-2 j-2 k} \otimes\left(\bar{Q}^{k} Q^{j} x\right)^{2} .
\end{aligned}
$$

When the last expression is examined, it is seen that all of the terms cancel except $u^{i+1} \otimes x$, if $i$ is odd or $i=0$, and $u^{i+1} \otimes x^{2}+u \otimes\left(Q^{a} x\right)^{2}$, if $i=2 a>0$ (remember, $\bar{Q} Q=1$ ).

Next recall the map $g: M^{*} \rightarrow P^{\infty}$ (which classifies the $Z_{2}$-covering $Z_{2} \rightarrow$ $\left.M \times M-\Delta \rightarrow M^{*}\right)$. It is easy to see, using the diagram

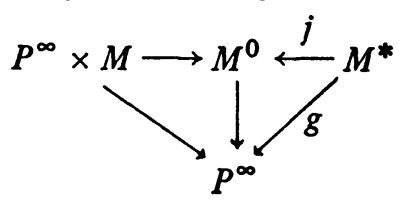

that $g^{*}(u)=j^{*}\left(u \otimes 1^{2}\right)$. In the basis of $H^{*}\left(M^{*}\right)$ that is being used, $j^{*}\left(u \otimes 1^{2}\right)$ $=\xi\left(u \otimes 1^{2}\right)=u \otimes 1^{2} \in H^{*}\left(M^{*}\right)$. The map $g$ introduces twisted cohomology for $M^{*}$. The next lemma computes the first twisted Bockstein, $\mathrm{Sq}^{1} g$, on $H^{*}\left(M^{*}\right)$.

Lemma 17. Let $x \in H^{r}(M)$. Then

$$
\mathrm{Sq}^{1} g\left(u^{2 i+1} \otimes x^{2}\right)=r u^{2 i+2} \otimes x^{2}+u^{2 i} \otimes\left(Q^{1} x\right)^{2},
$$

and

$$
\mathrm{Sq}^{1} g\left(u^{2 i} \otimes x^{2}\right)=(r+1) u^{2 i+1} \otimes x^{2}+u^{2 i-1} \otimes\left(Q^{1} x\right)^{2}+(r+i+1) u \otimes\left(Q^{i} x\right)^{2}
$$

Proof. This is an immediate consequence of Lemmas 16,12 and 13 , and the result in [5] which shows that $\mathrm{Sq}^{1} g(\alpha)=\mathrm{Sq}^{1}(\alpha)+g^{*}(u) \cdot \alpha$ for $\alpha \in H^{*}\left(M^{*}\right)$.

This completes the results needed for the study of $H^{*}\left(M^{*}\right)$ done in $\$ 7$. 
The rest of this section deals with similar results for the space $U^{*}$. First, a description of $\mathrm{Sq}^{1}$ on $H^{*}\left(U^{*}\right)$ is obtained.

LEMMA 18. Let $x \in H^{r}(M)$ and let $a \leqslant m-1$. Then in $H^{*}\left(U^{*}\right)$,

$$
\mathrm{Sq}^{1}\left(u^{a} \otimes x\right)=a u^{a+1} \otimes x+u^{a} \otimes Q^{1} x
$$

Proof. If $\gamma \in H^{*}\left(U^{*}\right)$, then the squaring operations on $\gamma$ are defined by $\mathrm{Sq}^{i} \gamma=\bar{\xi} \mathrm{Sq}^{i \bar{\xi}^{-1}}(\gamma)$. Thus

$$
\begin{aligned}
\mathrm{Sq}^{1}\left(u^{a} \otimes x\right)= & \bar{\xi} \mathrm{Sq}^{1} \xi^{-1}\left(u^{a} \otimes x\right)=\bar{\xi}\left(\mathrm{Sq}^{1} \sum_{i=0}^{a} u^{a-i} \otimes x w_{i}\right) \\
= & \bar{\xi}\left(\sum_{i=0}^{a}(a-i) u^{a+1-i} \otimes x w_{i}+\sum_{i=0}^{a} u^{a-i} \otimes \mathrm{Sq}^{1}\left(x w_{i}\right)\right) \\
= & \sum_{i=0}^{a} \sum_{k=0}^{a+1-i}(a-i) u^{a+1-i-k} \otimes \bar{w}_{k} x w_{i} \\
& +\sum_{i=0}^{a} \sum_{k=0}^{a-i} u^{a-i-k} \otimes \bar{w}_{k} \mathrm{Sq}^{1}\left(x w_{i}\right) .
\end{aligned}
$$

Now let $C_{s}$ be the sum of all elements in the last expression of the form $u^{a+1-s}$ $\otimes y$. Then $C_{0}=a u^{a+1} \otimes x$ and

$$
C_{1}=u^{a} \otimes \mathrm{Sq}^{1} x+(a-1) u^{a} \otimes x w_{1}+a u^{a} \otimes \bar{w}_{1} x=u^{a} \otimes Q^{1} x .
$$

If $s \geqslant 2$,

$$
\begin{aligned}
C_{s}= & \sum_{i=0}^{s}(a-i) u^{a+1-s} \otimes \bar{w}_{s-i} w_{t} x+\sum_{k=0}^{s-1} u^{a+1-s} \otimes \bar{w}_{k} \mathrm{Sq}^{1}\left(x w_{s-1-k}\right) \\
(*)= & \sum_{i=0}^{s}(a-i) u^{a+1-s} \otimes \bar{w}_{s-i} w_{i} x+\sum_{k=0}^{s-1} u^{a+1-s} \otimes \bar{w}_{k} w_{s-1-k} \mathrm{Sq}^{1} x \\
& +\sum_{k=0}^{s-1}(s-k) u^{a+1-s} \otimes \bar{w}_{k} w_{s-k} x+\sum_{k=0}^{s-1} u^{a+1-s} \otimes \bar{w}_{k} w_{s-1-k}\left(w_{1} x\right) .
\end{aligned}
$$

(Note: This uses $\mathrm{Sq}^{1} w_{j}=w_{1} w_{j}+(j+1) w_{j+1}$.) The second and fourth sums in the last expression are zero because $w \bar{w}=1$; the third sum is the same as $\Sigma_{j=1}^{s} j u^{a+1-s} \otimes \bar{w}_{s-j} w_{j} x$. When the last expression is substituted in (*), you get $C_{s}=\Sigma_{i=0}^{s} a u^{a+1-s} \otimes \bar{w}_{s-i} w_{i} x$ which is zero (again because $\bar{w} w=1$ ).

The composite $U^{*} \rightarrow M^{*} \stackrel{g}{\rightarrow} P^{\infty}$ is also called $g$. The final thing studied in this section is multiplication in $H^{*}\left(U^{*}\right)$ by $g^{*}(u)$ and the resulting formula for $\mathrm{Sq}^{1} g$.

LEMmA 19. Let $x \in H^{r}(M)$ and let $u^{a} \otimes x$ represent an element in $H^{*}\left(U^{*}\right)$ with $m-1 \geqslant a \geqslant m-r$. Then $\bar{\xi}\left(g^{*} u\right) \cdot u^{a} \otimes x=u^{a+1} \otimes x$. 
Proof. Products in $H^{*}\left(U^{*}\right)$ are defined the same way as in $H^{*}\left(M^{*}\right)$; thus the lemma follows by expanding the definition, collecting terms and using $\bar{w} w=1$ as in the above lemmas.

LEMMA 20. Let $x \in H^{r}(M)$ and consider $u^{a} \otimes x \in H^{*}\left(U^{*}\right)$ with $a+r \geqslant m$. Then $\mathrm{Sq}^{1} g\left(u^{a} \otimes x\right)=(a+1) u^{a+1} \otimes x+u^{a} \otimes Q^{1} x$.

Proof. This is a consequence of Lemmas 18 and 19 and [5].

The following is a summary of the important formulas obtained in this section $\left(x \in H^{r}(M)\right)$ :

$$
\begin{aligned}
& \text { (in } H^{*}\left(M^{*}\right) \text { ) } \\
& \operatorname{Sq}^{1}\left(u^{2 a+1} \otimes x^{2}\right)= \begin{cases}(r+1) u^{2 a+2} \otimes x^{2}+u^{2 a} \otimes\left(Q^{1} x\right)^{2}, & a>0, \\
(r+1) u^{2} \otimes x^{2}, & a=0,\end{cases} \\
& \mathrm{Sq}^{1}\left(u^{2 a} \otimes x^{2}\right)=\left\{\begin{array}{rr}
r u^{2 a+1} \otimes x^{2}+u^{2 a-1} \otimes\left(Q^{1} x\right)^{2} \\
+(r+a) u \otimes\left(Q^{a} x\right)^{2}, & a>0, \\
r u \otimes x^{2}+\mathrm{Sq}^{1} x \otimes x+x \otimes \mathrm{Sq}^{1} x, & a=0,
\end{array}\right.
\end{aligned}
$$

$u \otimes 1^{2} \cdot u^{2 a+1} \otimes x^{2}=u^{2 a+2} \otimes x^{2}$,

$u \otimes 1^{2} \cdot u^{2 a} \otimes x^{2}= \begin{cases}u^{2 a+1} \otimes x^{2}+u \otimes\left(Q^{a} x\right)^{2}, & a>0, \\ u \otimes x^{2}, & a=0,\end{cases}$

$\mathrm{Sq}^{1} g\left(u^{2 a+1} \otimes x^{2}\right)= \begin{cases}r u^{2 a+2} \otimes x^{2}+u^{2 a} \otimes\left(Q^{1} x\right)^{2}, & a>0, \\ r u^{2} \otimes x^{2}, & a=0,\end{cases}$

$$
\mathrm{Sq}^{1} g\left(u^{2 a} \otimes x^{2}\right)=\left\{\begin{aligned}
&(r+1) u^{2 a+1} \otimes x^{2}+u^{2 a-1} \otimes\left(Q^{1} x\right)^{2} \\
&+(r+a+1) u \otimes\left(Q^{a} x\right)^{2}, a>0, \\
&(r+1) u \otimes x^{2}+\mathrm{Sq}^{1} x \otimes x+x \otimes \mathrm{Sq}^{1} x, a=0,
\end{aligned}\right.
$$

(in $H^{*}\left(U^{*}\right)$ )

$$
\begin{aligned}
& \mathrm{Sq}^{1}\left(u^{a} \otimes x\right)=a u^{a+1} \otimes x+u^{a} \otimes Q^{1} x, \\
& \bar{\xi}(u \otimes 1) \cdot u^{a} \otimes x=u^{a+1} \otimes x \\
& \text { if } a+r \geqslant m \text {, } \\
& \mathrm{Sq}^{1} g\left(u^{a} \otimes x\right)=(a+1) u^{a+1} \otimes x+u^{a} \otimes Q^{1} x \quad \text { if } a+r \geqslant m .
\end{aligned}
$$

6. This section contains a calculation of the Bockstein spectral sequences associated to the exact sequences $0 \rightarrow Z \rightarrow Z \rightarrow Z_{2} \rightarrow 0$ and $0 \rightarrow Z f \rightarrow Z f \rightarrow$ $Z_{2} \rightarrow 0\left(f: Z_{2} \rightarrow\right.$ Aut $Z$ is an isomorphism) for the space $X^{0}=S^{\infty} \times z_{2} X \times X$, with $X$ a finite C.W. complex. The spectral sequence corresponding to the second 
exact sequence will be referred to as the twisted Bockstein spectral sequence (tBSS) (the twisting arises from the natural projection $g: X^{0} \rightarrow P^{\infty}$ ) while the one corresponding to the first exact sequence will be called the untwisted or usual Bockstein spectral sequence (BSS). The $E_{r}$-term of the tBSS for $X^{0}$ is denoted $E_{r}\left(X^{0}, g\right)$ while the $E_{r}$-term of the BSS for $X^{0}$ is denoted $E_{r}\left(X^{0}\right)$. In both cases the first term $\left(E_{1}\right)$ is $H^{*}\left(X^{0} ; Z_{2}\right)$.

Observe that both $E_{r}\left(X^{0}\right)$ and $E_{r}\left(X^{0}, g\right)$ are (graded) $Z_{2}$ vector spaces. The idea is to give a basis for each of those vector spaces in terms of a basis for the Bockstein spectral sequence (BSS) of the space $X$ (associated with $0 \rightarrow Z \rightarrow Z \rightarrow$ $\left.Z_{2} \rightarrow 0\right)$. The $E_{r}$-term of the last spectral sequence is denoted $E_{r}(X)\left(E_{r}^{k}(X)\right.$ means elements of degree $k$ ), and the $r$ th boundary is denoted $B_{r}$. The $r$ th boundary of $E_{r}\left(X^{0}\right)$ is $\underline{B}_{r}$ and of $E_{r}\left(X^{0}, g\right)$ is $\underline{B} g_{r}$. A nonzero element $\alpha \in E_{r}^{k}(X)$ is thought of as an element in $H^{k}(X)$ which has $B_{j} \alpha=0$ for $j<r$, and which is not in the image of $B_{j}$ for $j<r$. Use of the reduction map $H^{*}(X ; Z) \rightarrow$ $H^{*}\left(X ; Z_{2}\right)$ allows a basis (consisting of homogeneous elements) of $H^{*}\left(X ; Z_{2}\right)$ to be chosen with the property that if $\alpha$ is a basis element then one of the following cases holds:

(a) $B_{s} \alpha$ is also a basis element for a unique $s \geqslant 1$.

(b) $\alpha=B_{s} \alpha^{\prime}$ for a unique $s \geqslant 1$ and a unique basis element $\alpha^{\prime}$.

(c) $\alpha$ is the reduction of a class of infinite order.

THEOREM 21. For $r \geqslant 3$,

$$
\begin{aligned}
& E_{r}\left(X^{0}\right)=\left\{1 \otimes \alpha_{2 i} \mid 0 \neq \alpha_{2 i} \in E_{r-1}^{2 i}(X) \text { and } B_{r-1} \alpha_{2 i}=0\right\} \\
& \oplus\left\{\alpha \otimes \beta+\beta \otimes \alpha \mid \alpha \neq \beta \in E_{r}(X)\right\} \\
& \\
& \oplus\left\{B_{r-1} \alpha_{2 i+1} \otimes \alpha_{2 i+1}+\alpha_{2 i+1} \otimes B_{r-1} \alpha_{2 i-1} \mid\right. \\
&\left.\quad B_{r-1} \alpha_{2 i+1} \neq 0 \text { and } 0 \neq \alpha_{2 i+1} \in E_{r-1}(X)\right\}
\end{aligned}
$$

Moreover,

$$
\underline{B}_{r}\left(1 \otimes \alpha_{2 i}^{2}\right)=B_{r} \alpha_{2 i}+\alpha_{2 i} \otimes B_{r} \alpha_{2 i}
$$

$\underline{B}_{r}(\alpha \otimes \beta+\beta \otimes \alpha)=B_{r} \alpha \otimes \beta+\beta \otimes B_{r} \beta+\alpha \otimes B_{r} \beta+B_{r} \beta \otimes \alpha$,

and

$$
\underline{B}_{r}\left(B_{r-1} \alpha_{2 i+1} \otimes \alpha_{2 i+1}+\alpha_{2 i+1} \otimes B_{r-1} \alpha_{2 i+1}\right)=1 \otimes\left(B_{r-1} \alpha_{2 i+1}\right)^{2} .
$$

(If $r=2$, the description of $E_{r}$ is slightly different; see step 2 of the proof.)

REMARK. This theorem should be interpreted as saying that the elements $1 \otimes \alpha_{2 i}^{2}, \alpha \otimes \beta+\beta \otimes \alpha$, and $B_{r-1} \alpha_{2 i+1} \otimes \alpha_{2 i+1}+\alpha_{2 i+1} \otimes B_{r-1} \alpha_{2 i+1}$ in $H^{*}\left(X^{0}\right)$ (with the restrictions given) are cycles under $\underline{B}_{j}$ for $j<r$ and are not boundaries under $\underline{B}_{j}$ for $j<r$, and that $\underline{B}_{r}$ has the description given in the theorem. Thus a $Z_{2}$-basis for $E_{r}\left(X^{0}\right)$ is obtained by restricting $\alpha_{2 i}, \alpha, \beta$, and $\alpha_{2 i+1}$ to a basis for $E_{r}(X)$ as described in the paragraph preceding the statement of the theorem. 
THEOREM 22. For $r \geqslant 3$,

$$
\begin{gathered}
E_{r}\left(X^{0}, g\right)=\left\{1 \otimes \alpha_{2 i+1} \mid B_{r-1} \alpha_{2 i+1}=0 \text { and } 0 \neq \alpha_{2 i+1} \in E_{r-1}(X)\right\} \\
\oplus\left\{\alpha \otimes \beta+\beta \otimes \alpha \mid \alpha \neq \beta \in E_{r}(X)\right\} \\
\oplus\left\{B_{r-1} \alpha_{2 i} \otimes \alpha_{2 i}+\alpha_{2 i} \otimes B_{r-1} \alpha_{2 i} \mid\right. \\
\left.0 \neq \alpha_{2 i} \in E_{r-1}(X) \text { and } B_{r-1} \alpha_{2 i} \neq 0\right\}
\end{gathered}
$$

Moreover,

$$
B g_{r}\left(1 \otimes \alpha_{2 i+1}^{2}\right)=B_{r} \alpha_{2 i+1} \otimes \alpha_{2 i+1}+\alpha_{2 i+1} \otimes B_{r} \alpha_{2 i+1},
$$

$B g_{r}(\alpha \otimes \beta+\beta \otimes \alpha)=B_{r} \alpha \otimes \beta+\beta \otimes B_{r} \alpha+\alpha \otimes B_{r} \beta+B_{r} \beta \otimes \alpha$,

and

$$
\underline{B g}_{r}\left(B_{r-1} \alpha_{2 i} \otimes \alpha_{2 i}+\alpha_{2 i} \otimes B_{r-1} \alpha_{2 i}\right)=1 \otimes\left(B_{r-1} \alpha_{2 i}\right)^{2} \text {. }
$$

(Theorem 22 is interpreted in the same way as Theorem 21.)

Proof of Theorem 21. Step 1. Recall $C_{*}\left(X^{0} ; Z\right)=W_{*} \otimes_{Z(\pi)}$ $C_{*}(X \times X ; Z)$ where $\pi=Z_{2}=\{1, T\}$ and $W_{*}$ is the $Z(\pi)$-free resolution of the trivial $\pi$-module, $Z$, with $W_{n}=e_{n} \cdot Z(\pi)$ and $d\left(e_{n}\right)=\left(1+(-1)^{n} T\right) e_{n-1}$. Thus

$$
\begin{aligned}
C^{*}\left(X^{0} ; Z\right) & =\operatorname{hom}_{Z}\left(W_{*} \otimes_{Z(\pi)} C_{*}(X \times X ; Z) ; Z\right) \\
& =\operatorname{hom}_{Z(\pi)}\left(W_{*} ; C^{*}(X \times X ; Z)\right) \\
& =\operatorname{hom}_{Z(\pi)}\left(W_{*}, C^{*}(X ; Z) \otimes C^{*}(X ; Z)\right) .
\end{aligned}
$$

Next recall that if $f \in \operatorname{hom}\left(N_{*} ; P^{*}\right)\left(\left(N_{*}, d\right)\right.$ is a chain complex and $\left(P^{*}, \bar{d}\right)$ is a cochain complex) is an element of degree $n$, then $f=\Sigma f_{i}$ with $f_{i}: N_{i} \rightarrow P^{n-i}$ and $\delta f=\Sigma \delta f_{i}$ where $\delta f_{i}=f_{i} d+(-1)^{i} \bar{d} f_{i}$. Thus an element

$$
f_{i} \in \operatorname{hom}_{Z(\pi)}^{n}\left(W_{*} ; C^{*}(X) \otimes C^{*}(X)\right)
$$

can be written $f_{i}=\Sigma_{k} e_{i} \otimes a_{j, k} \otimes c_{n-i-j, k}$ with the convention that

$$
f_{i}\left(e_{i}\right)=\sum_{k} a_{j, k} \otimes c_{n-i-j, k}\left(a_{j, k} \in C^{j}(X ; Z) \text { and } c_{n-i-j, k} \in C^{n-i-j}(X ; Z)\right) \text {. }
$$

Using that notation the calculation of $\delta f_{i}$ is the following:

$$
\begin{aligned}
\delta f_{i}= & \sum_{k} e_{i+1} \otimes a_{j, k} \otimes c_{n-i-j, k} \\
& +(-1)^{i+1} \sum_{k}(-1)^{j(n-i-j)} e_{i+1} \otimes c_{n-i-j, k} \otimes a_{j, k} \\
& +(-1)^{i} \sum_{k} e_{i} \otimes \delta a_{j, k} \otimes c_{n-i-j, k} \\
& +(-1)^{i} \sum_{k}(-1)^{j} e_{i} \otimes a_{j, k} \otimes \delta c_{n-i-j, k} .
\end{aligned}
$$

The signs in this expression arise as follows: $(-1)^{i+1}$ comes from $d e_{i+1}=e_{i}+$ $(-1)^{i+1} T e_{i},(-1)^{j(n-i-j)}$ comes from $T e_{i} \otimes a \otimes c=e_{i} \otimes T(a \otimes c)=$ $(-1)^{j(n-i-j)} e_{i} \otimes c \otimes a,(-1)^{i}$ in the last two terms comes from $\delta f_{i}=f_{i} d+$ 
$(-1)^{i} \bar{d} f_{i}$, and $(-1)^{j}$ comes from $\delta(a \otimes c)=\delta a \otimes c+(-1)^{|a|} a \otimes \delta c(|a|$ is the degree of $a$ ).

If $\alpha$ is a homogeneous element in $H^{*}(X ; Z)$, let $a$ denote an integral cochain whose mod 2 reduction, $a^{\prime}$, is a representative for $\alpha$. Also let $\delta a=2^{s} a^{\prime \prime}$ where $s \geqslant 1(s=\infty$ means $\delta a=0)$ and $a^{\prime \prime}$ is an integral cochain whose mod 2 reduction is a representative for $B_{s} \alpha\left(c, c^{\prime}\right.$, and $c^{\prime \prime}$ have similar meanings for a homogeneous element $\gamma$ in $\left.H^{*}\left(X ; Z_{2}\right)\right)$. With this notation it is clear that $e_{i} \otimes a \otimes a$ and $e_{0} \otimes$ $a \otimes c+e_{0} \otimes c \otimes a$ are integral cochains representing $u^{i} \otimes \alpha^{2}$ and $\alpha \otimes \gamma+\gamma$ $\otimes \alpha$. Let $\alpha \in H^{r}\left(X ; Z_{2}\right)$, then

$$
\begin{aligned}
\delta\left(e_{i} \otimes a \otimes a\right)= & e_{i+1} \otimes a \otimes a+(-1)^{i+1+r^{2}} e_{i+1} \otimes a \otimes a \\
& +(-1)^{i} e_{i} \otimes 2^{s} a^{\prime \prime} \otimes a+(-1)^{i+r} e_{i} \otimes a \otimes 2^{s} a^{\prime \prime} .
\end{aligned}
$$

The sum of the first two terms in the last expression is 0 if $i+1+r^{2}=i+1+r$ is odd, and is 2 if $i+1+r$ is even. In either event $\delta\left(e_{i} \otimes a \otimes a\right)$ is divisible by 2 , and

$$
\left(1 / 2 \cdot \delta\left(e_{i} \otimes a \otimes a\right)\right)^{\prime}=\left\{\begin{array}{r}
e_{i+1} \otimes a^{\prime} \otimes a^{\prime}+e_{i} \otimes 2^{s-1} a^{\prime \prime} \otimes a^{\prime} \\
+e_{i} \otimes a^{\prime} \otimes 2^{s-1} a^{\prime \prime} \text { if } i+1+r \text { is even; } \\
e_{i} \otimes 2^{s-1} a^{\prime \prime} \otimes a^{\prime}+e_{i} \otimes a^{\prime} \otimes 2^{s-1} a^{\prime \prime} \\
\text { if } i+1+r \text { is odd. }
\end{array}\right.
$$

If $s>1$, the last two terms are zero, while if $s=1$ and $i>0$, a calculation shows $\delta\left(e_{i-1} \otimes a^{\prime \prime} \otimes a^{\prime}\right)=e_{i} \otimes a^{\prime \prime} \otimes a^{\prime}+e_{i} \otimes a^{\prime} \otimes a^{\prime \prime}$ (remember this takes place in $Z_{2}$, and $\delta a^{\prime}=0 \bmod 2$ ). Thus

$$
\mathrm{Sq}^{1}\left(u^{i} \otimes \alpha^{2}\right)= \begin{cases}(i+r) u^{i+1} \otimes \alpha^{2} & \text { if } \alpha \in H^{r}\left(X ; Z_{2}\right) \text { and } i>0, \\ r u \otimes \alpha^{2}+\mathrm{Sq}^{1} \alpha \otimes \alpha+\alpha \otimes \mathrm{Sq}^{1} \alpha, & i=0 .\end{cases}
$$

(Note: this is the proof of Lemma 11 of $\S 5$.)

Next consider the element $B_{s} \alpha \otimes \alpha+\alpha \otimes B_{s} \alpha$ where deg $\alpha=2 j-1 . b=$ $e_{0} \otimes a^{\prime \prime} \otimes a+e_{0} \otimes a \otimes a^{\prime \prime}$ is an integral cochain representing it (note $\operatorname{deg} a^{\prime \prime}=$ $2 j)$, and

$$
\begin{aligned}
\delta(b)= & e_{1} \otimes a^{\prime \prime} \otimes a+(-1)(-1)^{2 j(2 j+1)} e_{1} \otimes a \otimes a^{\prime \prime} \\
& +(-1)^{2 j} e_{0} \otimes a^{\prime \prime} \otimes \delta a+e_{1} \otimes a \otimes a^{\prime \prime} \\
& +(-1)(-1)^{(2 j-1)(2 j)} e_{1} \otimes a^{\prime \prime} \otimes a+e_{0} \otimes \delta a \otimes a^{\prime \prime} \\
= & e_{0} \otimes a^{\prime \prime} \otimes \delta a+e_{0} \otimes \delta a \otimes a^{\prime \prime} \\
= & e_{0} \otimes a^{\prime \prime} \otimes 2^{s} a^{\prime \prime}+e_{0} \otimes 2^{s} a^{\prime \prime} \otimes a^{\prime \prime} \\
= & 2^{s+1} e_{0} \otimes a^{\prime \prime} \otimes a^{\prime \prime} .
\end{aligned}
$$


Thus

$$
\underline{B}_{s+1}\left(B_{s} \alpha \otimes \alpha+\alpha \otimes B_{s} \alpha\right)=1 \otimes\left(B_{s} \alpha\right)^{2} \quad \text { if } \operatorname{deg} \alpha \text { is odd. }
$$

Similar calculations show that if $0 \neq \alpha \in E_{s}(X)$, and $0 \neq \gamma \in E_{t}(X)$, and $s \leqslant t$, then $0 \neq \alpha \otimes \gamma+\gamma \otimes \alpha \in E_{s}\left(X^{0}\right)$ and that

(3) $\underline{B}_{s}(\alpha \otimes \gamma+\gamma \otimes \alpha)=\left\{\begin{array}{l}B_{s} \alpha \otimes \gamma+\gamma \otimes B_{s} \alpha+B_{s} \gamma \otimes \alpha+\alpha \otimes B_{s} \gamma \text { if } s=t, \\ B_{s} \alpha \otimes \gamma+\gamma \otimes B_{s} \alpha, \quad s<t,\end{array}\right.$ also,

$$
\underline{B}_{s}\left(1 \otimes \alpha^{2}\right)=B_{s} \alpha \otimes \alpha+\alpha \otimes B_{s} \alpha \quad \text { if } \operatorname{deg} \alpha \text { is even. }
$$

Step 2. Equation (1) shows that the elements $u^{i} \otimes \alpha^{2}$ with $i \geqslant 1$ pair off under $\underline{B}_{1}=\mathrm{Sq}^{1}$ with the exception of the elements $u \otimes \alpha^{2}$ with degree of $\alpha$ odd. Moreover,

$$
\underline{B}_{1}\left(1 \otimes \alpha_{2 i+1}^{2}\right)=u \otimes \alpha_{2 i+1}^{2}+\mathrm{Sq}^{1} \alpha \otimes \alpha+\alpha \otimes \mathrm{Sq}^{1} \alpha,
$$

and

$$
\underline{B}_{1}\left(1 \otimes \alpha_{2 i}^{2}\right)=\mathrm{Sq}^{1} \alpha_{2 i} \otimes \alpha_{2 i}+\alpha_{2 i} \otimes \mathrm{Sq}^{1} \alpha_{2 i} \text {. }
$$

Equation (3) shows that the elements $\alpha \otimes \gamma+\gamma \otimes \alpha$ with $\alpha \neq \gamma \in E_{2}(X)$ are all nonzero and distinct in $E_{2}\left(X^{0}\right)$. Each of the remaining elements, $\alpha \otimes \gamma+\gamma \otimes \alpha$, falls into one of the following five sets:
(1) $\mathrm{Sq}^{1} \alpha \neq 0, \quad \gamma=\mathrm{Sq}^{1} \gamma^{\prime}$,
(2) $\mathrm{Sq}^{1} \alpha \neq 0, \quad \gamma \neq \mathrm{Sq}^{1} \gamma^{\prime}, \quad$ but $\mathrm{Sq}^{1} \gamma=0$,
(3) $\mathrm{Sq}^{1} \alpha \neq 0, \quad \mathrm{Sq}^{1} \gamma \neq 0$,
(4) $\alpha=\mathrm{Sq}^{1} \alpha^{\prime}, \quad \gamma=\mathrm{Sq}^{1} \gamma^{\prime}$,
(5) $\alpha=\mathrm{Sq}^{1} \alpha^{\prime}, \quad \gamma \neq \mathrm{Sq}^{1} \gamma^{\prime}, \quad$ but $\mathrm{Sq}^{1} \gamma=0$.

Now the elements in sets (2) and (5) pair off under $\underline{B}_{1}$. Also, each element in set (4) is hit (by $\underline{B}_{1}$ ) by two elements from set (1), and the sum of those elements is hit (by $\underline{B}_{1}$ ) by one element in set (3). Thus all the elements in those sets are "killed" in passing from $E_{1}$ to $E_{2}$. The conclusion is that $E_{2}$ has the following form:

$$
\begin{aligned}
E_{2}\left(X^{0}\right)= & \left\{u \otimes \alpha^{2}=\mathrm{Sq}^{1} \alpha \otimes \alpha+\alpha \otimes \mathrm{Sq}^{1} \alpha \mid \mathrm{Sq}^{1} \alpha \neq 0, \text { and deg } \alpha \text { is odd }\right\} \\
& \oplus\left\{1 \otimes \alpha_{2 i}^{2} \mid \mathrm{Sq}^{1} \alpha_{2 i}=0\right\} \oplus\left\{\alpha \otimes \gamma+\gamma \otimes \alpha \mid \alpha \neq \gamma ; \alpha, \gamma \in E_{2}(X)\right\} .
\end{aligned}
$$

(This is the description promised in the statement of Theorem 21.)

Step 3. Induction is now used to establish the theorem for arbitrary $r$. The things needed are equations (2) and (4), and a decomposition similar to that in Step 2. The details are easy and so are omitted. 
The proof of Theorem 22 uses a result due to Eilenberg [2, p. 412] which shows that $H^{*}\left(X^{0}, Z g\right)$ is the homology of the chain complex

$$
\operatorname{hom}_{\pi}\left(C_{*}\left(S^{\infty} \times X \times X ; Z\right), Z\right)
$$

where $Z$ has the nontrivial $\pi$-action given by $T(1)=-1$. Use of this fact makes it an easy matter to translate the proof of Theorem 21 into a proof of Theorem 22, so the details are omitted.

7. This section contains some general results on the structure of $E_{r}(Y)$ and $E_{r}(Y, g)$ for $Y=M^{*}$ or $U^{*}$ and certain manifolds $M$. For $M^{*}$ the idea is to study $E_{2}\left(M^{*}\right)\left(E_{2}\left(M^{*}, g\right)\right)$ and see when the map $j^{*}: H^{*}\left(M^{0}\right) \rightarrow H^{*}\left(M^{*}\right)$ induces an isomorphism $E_{2}\left(M^{0}\right) \rightarrow E_{2}\left(M^{*}\right)\left(E_{2}\left(M^{0}, g\right) \rightarrow E_{2}\left(M^{*}, g\right)\right)$. Thus the first step is to compute $E_{2}\left(M^{*}\right)$ and $E_{2}\left(M^{*}, g\right)$. The untwisted situation will be treated here. The twisted case is treated the same way; the only difference being the definition of $\mathrm{Sq}^{1} g$ on $H^{*}\left(M^{*}\right)$. Similar results for the space $U^{*}$ are obtained.

Note. In what follows, $E_{r}\left(M, Q^{1}\right)$ denotes the Bockstein spectral sequence of the manifold $M$ associated to the exact sequence $0 \rightarrow Z f \rightarrow Z f \rightarrow Z_{2} \rightarrow 0$, where $f: Z_{2} \rightarrow$ Aut $Z$ is an isomorphism, and the twisting of $M$ is given by $w_{1}: M \rightarrow K\left(Z_{2} ; 1\right)$. Since $Q^{1}(x)=\mathrm{Sq}^{1}(x)+x \cdot w_{1}$ for $x \in H^{*}(M)$, [5] shows that $Q^{1}$ is the first Bockstein in the spectral sequence. The spectral sequence is "related" to the twisted (by $\left.w_{1}\right)$ integer cohomology of $M, H^{*}\left(M ; Z w_{1}\right)$.

First, consider the following three subgroups of $H^{*}\left(M^{0}\right)\left(x, y \in H^{*}(M)\right.$ are homogeneous elements and $|x|$ is the degree of $x$ ).

$S_{1}$ in degree $2 m-j$ is generated by $\left\{u^{i} \otimes x^{2} \mid j \geqslant i>0\right.$, or $i=0$ and $|x|$ is odd $\}$

$\cup\left\{\mathrm{Sq}^{1} x \otimes x+x \otimes \mathrm{Sq}^{1} x|| x \mid\right.$ is odd $\} \cup\left\{1 \otimes\left(\mathrm{Sq}^{1} x\right)^{2}|| x \mid\right.$ is odd $\}$.

$S_{2}$ is generated by $\left\{x \otimes y+y \otimes x \mid x \neq y\right.$ and $x \neq \operatorname{Sq}^{1} y$ with $|y|$ odd $\}$ $\left\{1 \otimes x^{2}|| x \mid\right.$ is even and $\left.x \neq \operatorname{Sq}^{1} y\right\}$.

$S$ is generated by $\left\{u^{k} \otimes x^{2}+x \otimes 1 \cdot \delta_{2} \mid x \in H^{m-k}(M)\right\}$. Clearly $S$ is a subgroup of $S_{1} \oplus S_{2}$, and there is an exact sequence

$$
0 \rightarrow S \stackrel{i}{\longrightarrow} S_{1} \oplus S_{2} \stackrel{r}{\longrightarrow} S_{1} \oplus S_{2} / S \rightarrow 0 .
$$

Moreover, Yo's results show that $S_{1} \oplus S_{2} / S \simeq H^{*}\left(M^{*}\right)$.

Next, define an operator $\mathrm{Sq}^{1}$ on $S_{1} \oplus S_{2}$ as follows $\left(x \in H^{r}(M)\right)$ :

$$
\mathrm{Sq}^{1}\left(u^{i} \otimes x^{2}\right)= \begin{cases}(r+1) u^{i+1} \otimes x^{2}+u^{i-1} \otimes\left(Q^{1} x\right)^{2}, & i \text { odd }>1, \\ r u^{i+1} \otimes x^{2}+u^{i-1} \otimes\left(Q^{1} x\right)^{2}+(r+a) u \otimes\left(Q^{a} x\right)^{2}, & i=2 a>0 \\ (r+1) u^{2} \otimes x^{2}, & i=1, \\ r u \otimes x^{2}+\mathrm{Sq}^{1} x \otimes x+x \otimes \mathrm{Sq}^{1} x, & i=0 .\end{cases}
$$


$\mathrm{Sq}^{1}(x \otimes y+y \otimes x)$ is given by the Cartan formula. $S_{1}$ and $S_{2}$ were chosen so that $\mathrm{Sq}^{1}\left(S_{1}\right) \subset S_{1}$ and $\mathrm{Sq}^{1}\left(S_{2}\right) \subset S_{2}$. The following lemma shows that $\mathrm{Sq}^{1}(S) \subset S$.

LEMMA 23. $\mathrm{Sq}^{1}\left(u^{k} \otimes x^{2}+x \otimes 1 \cdot \delta_{2}\right)=u^{k-1} \otimes\left(Q^{1} x\right)^{2}+Q^{1} x \otimes 1 \cdot$ $\delta_{2}$ where $x \in H^{m-k}(M)$.

Proof. This is a calculation.

Case 1 ( $k$ odd). Note $2 m-k$ is odd, so $x \otimes 1 \cdot \delta_{2}$ contains no elements of the form $1 \otimes y^{2}$. The above definition of $\operatorname{Sq}^{1}$ shows $\operatorname{Sq}^{1}\left(u^{k} \otimes x^{2}\right)=u^{k-1}$ $\otimes\left(Q^{1} x\right)^{2}$, and

$$
\begin{aligned}
\mathrm{Sq}^{1}\left(x \otimes 1 \cdot \delta_{2}\right)= & \mathrm{Sq}^{1} x \otimes 1 \cdot \delta_{2}+x \otimes 1 \cdot \mathrm{Sq}^{1} \delta_{2} \\
= & \mathrm{Sq}^{1} x \otimes 1 \cdot \delta_{2}+x \otimes 1 \cdot w_{1} \otimes 1 \cdot \delta_{2} \\
& \quad(\text { by Lemma 14, §5) } \\
= & \left(\mathrm{Sq}^{1} x+x \cdot w_{1}\right) \otimes 1 \cdot \delta_{2}=Q^{1} x \otimes 1 \cdot \delta_{2} .
\end{aligned}
$$

Case $2(k=2 a)$. This time $2 m-k$ is even, so $x \otimes 1 \cdot \delta_{2}$ is the sum of elements $y \otimes z+z \otimes y$ plus the elements $1 \otimes\left(Q^{a} x\right)^{2}$ (by Lemma 15, §5). Now

$$
\mathrm{Sq}^{1}\left(u^{2 a} \otimes x^{2}\right)=u^{k-1} \otimes\left(Q^{1} x\right)^{2}+(m-k+a) u \otimes\left(Q^{a} x\right)^{2}
$$

Also,

$$
\begin{aligned}
\mathrm{Sq}^{1}\left(x \otimes 1 \cdot \delta_{2}\right) & =\mathrm{Sq}^{1} x \otimes 1 \cdot \delta_{2}+x \otimes 1 \cdot \mathrm{Sq}^{1} \delta_{2}+(m-k+a) u \otimes\left(Q^{a} x\right)^{2} \\
& =Q^{1} x \otimes 1 \cdot \delta_{2}+(m-k+a) u \otimes\left(Q^{a} x\right)^{2} .
\end{aligned}
$$

A consequence of Lemma 23 is that there is a map $S_{1} \oplus S_{2} / S \rightarrow S_{1} \oplus$ $S_{2} / S$ which is induced by $\mathrm{Sq}^{1}$ on $S_{1} \oplus S_{2}$ and which agrees with the topological $\mathrm{Sq}^{1}$ defined on $H^{*}\left(M^{*}\right)$. Moreover, since (1) is a short exact sequence of $\mathrm{Sq}^{1}$. modules, there is the following long exact sequence of homologies (i.e. $E_{2}\left(S_{1}\right) \simeq$ $H_{*}\left(S_{1} ; \mathrm{Sq}_{i_{*}}^{1}\right)$, etc.):

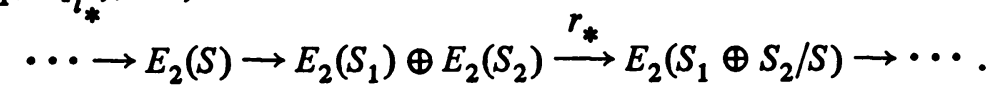

$E_{2}(S)$ and $E_{2}\left(S_{1}\right)$ are computed for certain manifolds in

LEMMA 24. $E_{2}(S)=\left\{u^{k} \otimes y^{2}+y \otimes 1 \cdot \delta_{2}|| y \mid=m-k, Q^{1} y=0\right.$ and $y \neq Q^{1}$ z, i.e. $\left.0 \neq y \in E_{2}\left(M ; Q^{1}\right)\right\}$.

If $m$ is odd, then

$$
\begin{aligned}
E_{2}^{2 m-4 c}\left(S_{1}\right)= & \left\{u^{4 c} \otimes y^{2}+1 \otimes\left(Q^{2 c} y\right)^{2}|| y \mid=m-4 c \text { and } 0 \neq y \in E_{2}\left(M ; Q^{1}\right)\right\} \\
E_{2}^{2 m-4 c-1}\left(S_{1}\right)= & \left\{u^{4 c+1} \otimes y^{2}|| y \mid=m-4 c-1 \text { and } 0 \neq y \in E_{2}\left(M ; Q^{1}\right)\right\} \\
E_{2}^{2 m-4 c-2}\left(S_{1}\right)= & \left\{u^{4 c+2} \otimes y^{2}|| y \mid=m-4 c-2 \text { and } 0 \neq y \in E_{2}\left(M ; Q^{1}\right)\right\} \\
& \oplus\left\{1 \otimes\left(\mathrm{Sq}^{1} x\right)^{2}|| x \mid=m-2 c-2\right\}
\end{aligned}
$$




$$
\begin{aligned}
E_{2}^{2 m-4 c-3}\left(S_{1}\right)= & \left\{u^{4 c+3} \otimes y^{2}|| y \mid=m-4 c-3 \text { and } 0 \neq y \in E_{2}\left(M ; Q^{1}\right)\right\} \\
& \oplus\left\{u \otimes x^{2}=\mathrm{Sq}^{1} x \otimes x+x \otimes \mathrm{Sq}^{1} x|| x \mid=m-2 c-2\right\}
\end{aligned}
$$

If $m$ is even $(m=2 a)$ then

$$
\begin{aligned}
E_{2}^{2 m-4 c}\left(S_{1}\right) & =\left\{1 \otimes\left(\mathrm{Sq}^{1} x\right)^{2}|| x \mid=m-2 c-1\right\}, \\
E_{2}^{2 m-4 c-1}\left(S_{1}\right) & =\left\{\mathrm{Sq}^{1} x \otimes x+x \otimes \mathrm{Sq}^{1} x=u \otimes x^{2}|| x \mid=m-2 c-1\right\}, \\
E_{2}^{2 m-4 c-2}\left(S_{1}\right) & =0, \\
E_{2}^{2 m-4 c-3}\left(S_{1}\right) & =0 .
\end{aligned}
$$

Proof. The statement concerning $S$ is obvious from the definition of $\mathrm{Sq}^{1}$ given above. The other two statements involve writing down a basis for $S_{1}$ in each dimension and observing that the $\mathrm{Sq}^{1}$ operator is periodic with period 4 . Thus induction can be used. The process is long, not illuminating, and omitted.

(Note: for the description with $m$ odd, the following lemma must be used!)

Lemma 25. If $m$ is odd, $y \in H^{m-4 a}(M)$, and $Q^{1} y=0$, then $\mathrm{Sq}^{1} Q^{2 a} y=0$. Also if $m$ is odd, $y \in H^{m-4 a-2}(M)$, and $Q^{1} y=0$, then $\operatorname{Sq}^{1} Q^{2 a+1} y=0$.

Proof. The second statement is obvious, since $Q^{2 a+1} y=Q^{2 a} Q^{1} y=0$. The proof of the first statement is more difficult. It suffices to show that $0=$ $\left\langle z \cdot \mathrm{Sq}^{1} Q^{2 a} y, M\right\rangle$ for all $z \in H^{2 a-1}(M)$. Now

$$
\begin{aligned}
\left\langle z \cdot \mathrm{Sq}^{1} Q^{2 a} y, M\right\rangle & =\left\langle\mathrm{Sq}^{1} z \cdot Q^{2 a} y, M\right\rangle+\left\langle\mathrm{Sq}^{1}\left(z \cdot Q^{2 a} y\right), M\right\rangle \\
& =\left\langle\mathrm{Sq}^{2 a}\left(\mathrm{Sq}^{1} z\right) \cdot y, M\right\rangle+\left\langle w_{1} z \cdot Q^{2 a} y, M\right\rangle \\
& =\left\langle\left(\mathrm{Sq}^{1} z\right)^{2} \cdot y, M\right\rangle+\left\langle\mathrm{Sq}^{2 a}\left(w_{1} z\right) \cdot y, M\right\rangle .
\end{aligned}
$$

But

$$
\begin{aligned}
\left(\mathrm{Sq}^{1} z\right)^{2} \cdot y & =\mathrm{Sq}^{1}\left(z \mathrm{Sq}^{1} z\right) \cdot y=\mathrm{Sq}^{1}\left(z \mathrm{Sq}^{1} z \cdot y\right)+z \mathrm{Sq}^{1} z \mathrm{Sq}^{1} y \\
& =w_{1} z \mathrm{Sq}^{1} z \cdot y+z \mathrm{Sq}^{1} z \cdot w_{1} y=0 .
\end{aligned}
$$

This uses $Q^{1}=\mathrm{Sq}^{1}+w_{1}, Q^{1}\left(z \mathrm{Sq}^{1} z \cdot y\right)=0$ (since $Q^{1} H^{m-1}(M)=0$ ), and $Q^{1} y=0$. Also,

$$
\mathrm{Sq}^{2 a}\left(w_{1} z\right) \cdot y=w_{1}^{2} z^{2} \cdot y=w_{1} z^{2} w_{1} \cdot y=w_{1} z^{2} \operatorname{Sq}^{1} y=\operatorname{Sq}^{1}\left(z^{2} \operatorname{Sq}^{1} y\right)=0 .
$$

The last equality is a consequence of the Cartan formula (since $\mathrm{Sq}^{1}\left(z^{2}\right)=0$ ) and the next to last equality follows because $Q^{1}=\mathrm{Sq}^{1}+w_{1}$.

TheOREM 26. Let $M$ be an odd dimensional manifold. Then

$$
\begin{aligned}
E_{2}\left(M^{*}\right) \simeq & E_{2}\left(S_{2}\right) \oplus\left\{1 \otimes\left(\mathrm{Sq}^{1} x\right)^{2}|| x \mid=m-2 c-2\right\} \\
& \oplus\left\{u \otimes x^{2}=\mathrm{Sq}^{1} x \otimes x+x \otimes \mathrm{Sq}^{1} x|| x \mid=m-2 c-2\right\},
\end{aligned}
$$

and the map $j^{*}: H^{*}\left(M^{0}\right) \rightarrow H^{*}\left(M^{*}\right)$ induces isomorphisms $E_{r}\left(M^{0}\right) \rightarrow E_{r}\left(M^{*}\right)$ for $r \geqslant 2$. 
Proof. The hypotheses imply that the map $i_{*}$ (in sequence (2)) is a monomorphism whose cokernel is the set of elements listed in the theorem. But that set of elements is precisely the set $E_{2}\left(M^{0}\right)$ calculated in Theorem $21, \S 6$. Also the map $r$ (in sequence (1)) is just $j^{*} \mid S_{1} \oplus S_{2}$, so $r_{*}$ corresponds to the map $E_{2}\left(M^{0}\right) \rightarrow E_{2}\left(M^{*}\right)$ induced by $j^{*}$. Since this is an isomorphism for $r=2$, it will be for all $r \geqslant 2$ as well.

For even dimensional manifolds (2) does not break into short exact sequences unless $E_{2}^{m-c}\left(M ; Q^{1}\right)=0$, all $c \geqslant 1$. Thus in general there is not much that can be said about $E_{r}\left(M^{*}\right)$ (at least if the above method is used).

The study of $E_{r}\left(M^{*}, g\right)$ is in "some sense dual" to the study of $E_{r}\left(M^{*}\right)$. Results true for $E_{r}\left(M^{*}\right)$ and odd (even) dimensional manifolds are true for $E_{r}\left(M^{*}, g\right)$ and even (odd) dimensional manifolds. In what follows I have indicated the changes necessary in the above discussion to obtain Theorem 26 for $E_{r}\left(M^{*}, g\right)$.

First, let $S_{1} g$ and $S_{2} g$ be the subgroups of $H^{*}\left(M^{0}\right)$ generated by the following sets:

$S_{1} g($ in degree $2 m-j)=\left\{u^{i} \otimes x^{2} \mid j \geqslant i>0\right\} \cup\left\{1 \otimes x^{2}|| x \mid\right.$ is even $\}$

$\cup\left\{1 \otimes\left(\mathrm{Sq}^{1} x\right)^{2}|| x \mid\right.$ is even $\} \cup\left\{\mathrm{Sq}^{1} x \otimes x+x \otimes \mathrm{Sq}^{1} x|| x \mid\right.$ is even $\}$

$S_{2} g=\left\{x \otimes y+y \otimes x \mid x \neq y\right.$, and $x \neq \operatorname{Sq}^{1} y$ with $|y|$ even $\}$

$U\left\{1 \otimes x^{2}|| x \mid\right.$ is odd and $\left.x \neq \mathrm{Sq}^{1} y\right\}$.

The subset $S$ remains the same. If $x \in H^{r}(M)$, the $\mathrm{Sq}^{1} g$ is defined by

$$
\mathrm{Sq}^{1} g\left(u^{i} \otimes x^{2}\right)=\left\{\begin{array}{cc}
r u^{i+1} \otimes x^{2}+u^{i-1} \otimes\left(Q^{1} x\right)^{2}, & i \text { odd }>1, \\
(r+1) u^{i+1} \otimes x^{2}+u^{i-1} \otimes\left(Q^{1} x\right)^{2} & \\
+(r+a+1) u \otimes\left(Q^{a} x\right)^{2}, & i=2 a>0 \\
r u^{2} \otimes x^{2}, & i=1, \\
(r+1) u \otimes x^{2}+\mathrm{Sq}^{1} x \otimes x+x \otimes \mathrm{Sq}^{1} x, & i=0,
\end{array}\right.
$$

The $g$-versions of Lemmas 1 and 2 are the following:

LEMMA 23-g. $\mathrm{Sq}^{1} g\left(u^{k} \otimes x^{2}+x \otimes 1 \cdot \delta_{2}\right)=u^{k-1} \otimes\left(Q^{1} x\right)^{2}+Q^{1} x \otimes$ $1 \cdot \delta_{2}$, for $x \in H^{m-k}(M)$.

Lemma 24-g. $E_{2}(S, g)=\left\{u^{k} \otimes y^{2}+y \otimes 1 \cdot \delta_{2} \| y \mid=m-k\right.$ and $0 \neq y \in$ $\left.E_{2}\left(M ; Q^{1}\right)\right\}$.

If $m$ is even, then

$$
\begin{gathered}
E_{2}^{2 m-4 c}\left(S_{1}, g\right)=\left\{u^{4 c} \otimes y^{2}+1 \otimes Q^{2 c} y^{2}|| y \mid=m-4 c\right. \\
\left.\quad \text { and } 0 \neq y \in E_{2}\left(M ; Q^{1}\right)\right\}, \\
E_{2}^{2 m-4 c-1}\left(S_{1}, g\right)=\left\{u^{4 c+1} \otimes y^{2}|| y \mid=m-4 c-1 \text { and } 0 \neq y \in E_{2}\left(M ; Q^{1}\right)\right\},
\end{gathered}
$$




$$
\begin{aligned}
& E_{2}^{2 m-4 c-2}\left(S_{1}, g\right)=\left\{u^{4 c+2} \otimes y^{2}|| y \mid=m-4 c-2 \text { and } 0 \neq y \in E_{2}\left(M ; Q^{1}\right)\right\} \\
& \oplus\left\{1 \otimes\left(\mathrm{Sq}^{1} x\right)^{2}|| x \mid=m-2 c-2\right\}, \\
& E_{2}^{2 m-4 c-3}\left(S_{1}, g\right)=\left\{u^{4 c+3} \otimes y^{2}|| y \mid=m-4 c-3 \text { and } 0 \neq y \in E_{2}\left(M ; Q^{1}\right)\right\} \\
& \oplus\left\{u \otimes x^{2}=\mathrm{Sq}^{1} x \otimes x+x \otimes \mathrm{Sq}^{1} x|| x \mid=m-2 c-2\right\} .
\end{aligned}
$$

If $m$ is odd, then

$$
\begin{aligned}
E_{2}^{2 m-4 c}\left(S_{1}, g\right) & =\left\{1 \otimes\left(\mathrm{Sq}^{1} x\right)^{2}|| x \mid=m-2 c-1\right\}, \\
E_{2}^{2 m-4 c-1}\left(S_{1}, g\right) & =\left\{\mathrm{Sq}^{1} x \otimes x+x \otimes \mathrm{Sq}^{1} x=u \otimes x^{2}|| x \mid=m-2 c-1\right\}, \\
E_{2}^{2 m-4 c-2}\left(S_{1}, g\right) & =0, \quad E_{2}^{2 m-4 c-3}\left(S_{1}, g\right)=0 .
\end{aligned}
$$

Finally, the following is the $g$-version of Theorem 1 .

THEOREM 26-g. Let $M$ be an even dimensional manifold. Then

$$
\begin{aligned}
E_{2}\left(M^{*}, g\right) \simeq & E_{2}\left(S_{2}, g\right) \oplus\left\{1 \otimes\left(\mathrm{Sq}^{1} x\right)^{2}|| x \mid=m-2 c-2\right\} \\
& \oplus\left\{\mathrm{Sq}^{1} x \otimes x+x \otimes \mathrm{Sq}^{1} x=u \otimes x^{2}|| x \mid=m-2 c-2\right\},
\end{aligned}
$$

and the map $j^{*}: H^{*}\left(M^{0}\right) \rightarrow H^{*}\left(M^{*}\right)$ induces an isomorphism $E_{r}\left(M^{0} ; g\right) \rightarrow$ $E_{r}\left(M^{*} ; g\right)$ for all $r \geqslant 2$.

The next two theorems contain general results about $E_{r}^{i}\left(U^{*}\right)$ and $E_{r}^{i}\left(U^{*}, g\right)$ (in dimensions $i>m$ ) for a manifold $M$. The results are not as hard to obtain as those dealing with $M^{*}$, partly because I have been unable to give a general description of the $E_{3}$-term when the $E_{2}$-term is nonzero. Fortunately, the $E_{2}$-term is zero half of the time.

TheOREM 27. Let $M$ be an odd dimensional manifold, and let $i<m$. Then $E_{2}^{2 m-i}\left(U^{*}\right)=0$.

Let $M$ be an even dimensional manifold, and let $i<m$. Then $E_{2}^{2 m-i}\left(U^{*}\right) \simeq$ $\left\{u^{m-1} \otimes y \mid 0 \neq y \in E_{2}^{m-i+1}\left(M ; Q^{1}\right)\right\}$. (Since $Q^{1}$ is the first Bockstein of the twisted $\left(\right.$ by $\left.w_{1}\right)$ integer cohomology of $M, E_{2}^{j}\left(M ; Q^{1}\right)=0$ if all the 2-torsion of $H^{j}\left(M ; Z w_{1}\right)$ is of order 2.)

Proof. This is proved by writing down Yo's basis for $H^{*}\left(U^{*}\right)$ and using the description of $\mathrm{Sq}^{1}$ given in $\S 5$. The details are tedious, but straightforward.

The next result deals with the twisted (by the map $g: U^{*} \rightarrow P$ ) cohomology of $U^{*}$ and is "dual" to Theorem 27.

THEOREM 27-g. Let $M$ be an even dimensional manifold, and let $i<m$. Then $E_{2}^{2 m-i}\left(U^{*}, g\right)=0$.

Let $M$ be an odd dimensional manifold, and let $i<m$. Then $E_{2}^{2 m-i}\left(U^{*}, g\right)$ $=\left\{u^{m-1} \otimes y \mid 0 \neq y \in E_{2}^{m-i+1}\left(M ; Q^{1}\right)\right\}$.

It is possible to obtain Theorem 27 for $m$ odd and Theorem 27-g for $m$ even 
by studying the Serre spectral sequence of the fibration $P^{m-1} \rightarrow U^{*} \rightarrow M$ [27]. The advantage of the approach here is that the map on cohomology induced by the inclusion $U^{*} \rightarrow M^{*}$ is readily computed and can be used to study the map $\operatorname{Emb}\left(M, R^{n}\right) \rightarrow \operatorname{Imm}\left(M, E^{n}\right)$.

8. The final thing needed to study the various embedding-immersion sets is a description of the $A^{i}$-operations. This section contains a summary of the appropriate formulas. Note: $[26, \S 6]$ contains formulas for $\mathrm{Sq}^{i}$ acting on $H^{*}\left(M^{*}\right)$; something is wrong though, because, for example, if you use them to compute $\mathrm{Sq}^{1} \cdot \mathrm{Sq}^{1}$, you do not get zero.

The first lemma concerns $\mathrm{Sq}^{2}$ on $H^{*}\left(M^{*}\right)$. A simple form for $\mathrm{Sq}^{2}$ has not been found. Fortunately, it is not necessary to compute $\mathrm{Sq}^{2}$ on very many elements of $H^{*}\left(M^{*}\right)$.

Lemma 28. Let $x \in H^{r}(M)$ and let $u^{a} \otimes x \in H^{*}\left(M^{*}\right)$. Then

$$
\begin{aligned}
& \mathrm{Sq}^{2}\left(u^{a} \otimes x^{2}\right)=\sum_{i=0}^{[a-1 / 2]} \sum_{j=0}^{[a+1-2 i / 2]} R_{a-2 i, r+i} u^{a+2-2 i-2 j} \otimes\left(\bar{Q}^{j} Q^{i} x\right)^{2} \\
& +\sum_{i=0}^{[a-1 / 2]} \sum_{j=0}^{[a-1-2 i / 2]} u^{a-2 i-2 j} \otimes\left(\bar{Q}^{\prime} \mathrm{Sq}^{1} Q^{i} x\right)^{2}
\end{aligned}
$$

where

$$
R_{b, c}=\left(\begin{array}{l}
b \\
2
\end{array}\right)+b c+\left(\begin{array}{l}
c \\
2
\end{array}\right) \in Z_{2}
$$

(Note: if $a=0, \mathrm{Sq}^{2} x \otimes x+\mathrm{Sq}^{2} x$ must be added to the above element.)

Proof. This is a consequence of the definition

$$
\mathrm{Sq}^{2}\left(u^{a} \otimes x^{2}\right)=\xi \mathrm{Sq}^{2} \xi^{-1}\left(u^{a} \otimes x^{2}\right)
$$

and

LEMma 29. Let $x \in H^{r}(M)$. In $H^{*}\left(M^{0}\right)$

$$
\mathrm{Sq}^{2}\left(u^{a} \otimes x^{2}\right)= \begin{cases}R_{a, r} u^{a+2} \otimes x^{2}+u^{a} \otimes\left(\mathrm{Sq}^{1} x\right)^{2}, & a>0, \\ R_{a, r} u^{a+2} \otimes x^{2}+u^{a} \otimes\left(\mathrm{Sq}^{1} x\right)^{2}+\mathrm{Sq}^{2} x \otimes x+x \otimes \mathrm{Sq}^{2} x, & a=0 .\end{cases}
$$

Proof. This lemma uses the diagram:

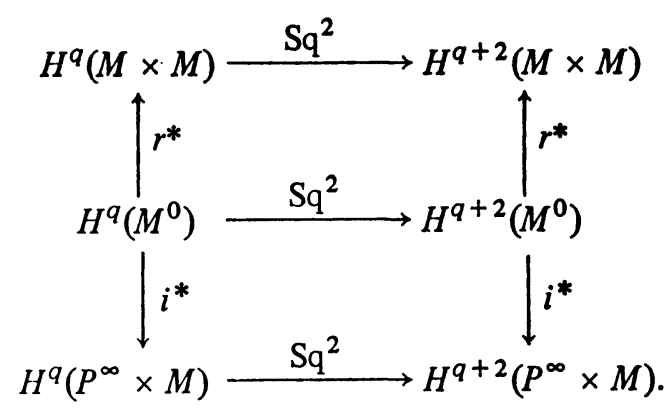


When $a=0$, it is straightforward to check that the above definition of $\mathrm{Sq}^{2}$ makes both squares commute. Since $i^{*}$ is $1-1$ on $H^{*}\left(P^{\infty}\right) \otimes D$ and $r^{*}$ is $1-1$ on $N$, $\mathrm{Sq}^{2}\left(1 \otimes x^{2}\right)$ must be as described. $\mathrm{Sq}^{2}\left(u^{a} \otimes x^{2}\right)$ (for $\left.a>0\right)$ is obtained by applying the Cartan formula to $u^{a} \otimes 1^{2} \cdot 1 \otimes x^{2}=u^{a} \otimes x^{2}$ and using the value of $\mathrm{Sq}^{1}\left(1 \otimes x^{2}\right)$ obtained in $\S 5$. (It takes a little work to obtain the coefficient $R_{a, r}$ in the lemma.)

Corollary 30. In $H^{*}\left(M^{*}\right) \mathrm{Sq}^{2}$ has the following description:

$$
\begin{aligned}
& u \otimes x_{m-2}^{2} \mapsto \begin{cases}u \otimes\left(\mathrm{Sq}^{1} x_{m-2}\right)^{2}, & m \equiv 1,2 \text { (4), } \\
u \otimes\left(w_{1} x_{m-2}\right)^{2}, & m \equiv 0,3 \text { (4), }\end{cases} \\
& u^{3} \otimes x_{m-3}^{2} \mapsto u \otimes\left(w_{1}^{2} x_{m-3}\right)^{2}, \\
& 1 \otimes x_{m-2}^{2} \mapsto\left(\begin{array}{c}
m-2 \\
2
\end{array}\right) u^{2} \otimes x_{m-2}^{2}+1 \otimes\left(\mathrm{Sq}^{1} x_{m-2}\right)^{2} \\
& +\mathrm{Sq}^{2} x_{m-2} \otimes x_{m-2}+x_{m-2} \otimes \mathrm{Sq}^{2} x_{m-2} \\
& u^{2} \otimes x_{m-3}^{2} \mapsto \begin{cases}u^{2} \otimes\left(\mathrm{Sq}^{1} x_{m-3}\right)^{2}, & m \equiv 1,2(4), \\
u^{2} \otimes\left(w_{1} x_{m-3}\right)^{2}, & m \equiv 0,3(4),\end{cases} \\
& u^{4} \otimes x_{m-4}^{2} \mapsto \begin{cases}u^{2} \otimes\left(w_{1}^{2} x_{m-4}\right)^{2}, & m \equiv 0,1 \text { (4), } \\
u^{2} \otimes\left(\mathrm{Sq}^{2} x_{m-4}+w_{1} \mathrm{Sq}^{1} x_{n-4}+w_{2} x_{m-4}\right)^{2}, & m \equiv 2,3 \text { (4). }\end{cases}
\end{aligned}
$$

In $H^{*}\left(M^{0}\right) \xi$ and $\xi^{-1}$ are each the identity on $u^{2} \otimes 1^{2}$. That means that multiplication by $g^{*}(u)^{2}=u^{2} \otimes 1^{2}=u \otimes 1^{2} \cdot u \otimes 1^{2}$ may be computed from the formula obtained in $\S 5$ for multiplication by $u \otimes 1^{2}$. That fact gives

Lemma 31. Let $x \in H^{r}(M)$. In $H^{*}\left(M^{*}\right)$ the following formulas hold:

$$
u^{2} \otimes 1^{2} \cdot u^{2 i+1} \otimes x^{2}=u^{2 i+3} \otimes x^{2}+u \otimes\left(Q^{i+1} x\right)^{2}
$$

and

$$
u^{2} \otimes 1^{2} \cdot u^{2 i} \otimes x^{2}= \begin{cases}u^{2 i+3} \otimes x^{2}+u^{2} \otimes\left(Q^{i} x\right)^{2}, & i>0, \\ u^{2} \otimes x, & i=0 .\end{cases}
$$

COROLlary 32. In $H^{*}\left(M^{*}\right)$ multiplication by $g^{*}(u)^{2}$ takes the following values:

$$
\begin{aligned}
& u \otimes x_{m-2}^{2} \stackrel{g^{*}(u)^{2} \cdot-}{\longrightarrow} u \otimes\left(Q^{1} x_{m-2}\right)^{2}, \quad u^{3} \otimes x_{m-3}^{2} \mapsto 0, \\
& 1 \otimes x_{m-2}^{2} \mapsto u^{2} \otimes x_{m-2}^{2}, \quad u^{2} \otimes x_{m-3}^{2} \mapsto u^{2} \otimes\left(Q^{1} x_{m-3}\right)^{2}, \\
& u^{4} \otimes x_{m-4}^{2} \mapsto u^{2} \otimes\left(Q^{2} x_{m-4}\right)^{2} .
\end{aligned}
$$

Yo computed $\mathrm{Sq}^{2}$ on $H^{*}\left(U^{*}\right)$ in $[26$, p. 1790]; his result is 
LEMMA 33. Let $x \in H^{r}(M)$ and let $m-1 \geqslant a$. Then in $H^{*}\left(U^{*}\right)$

$$
\mathrm{Sq}^{2}\left(u^{a} \otimes x\right)=\sum_{\substack{i=0, \ldots, a ; j=0,1,2 \\
k=0, \ldots, a+2-i-j}}\left(\begin{array}{c}
a-i \\
2-j
\end{array}\right) u^{a+2-i-j-k} \otimes \bar{w}_{k} \mathrm{Sq}^{j}\left(x \cdot w_{i}\right) \text {. }
$$

CoRollary 34. $\mathrm{Sq}^{2}$ on $H^{2 m-i}\left(U^{*}\right)($ for $i=3,4)$ has the following description:

$$
\begin{aligned}
u^{m-3} \otimes x_{m} & \stackrel{\mathrm{Sq}^{2}}{\mapsto}\left(\begin{array}{c}
m-3 \\
2
\end{array}\right) u^{m-1} \otimes x_{m}, \\
u^{m-2} \otimes x_{m-1} & \mapsto u^{m-1} \otimes \mathrm{Sq}^{1} x_{m-1}+(m-3) u^{m-1} \otimes Q^{1} x_{m-1}, \\
u^{m-1} \otimes x_{m-2} & \mapsto u^{m-1} \otimes w_{1}^{2} x_{m-2}, \\
u^{m-4} \otimes x_{m} & \mapsto\left(\begin{array}{c}
m-4 \\
2
\end{array}\right) u^{m-2} \otimes x_{m}, \\
u^{m-3} \otimes x_{m-1} & \mapsto\left(\begin{array}{c}
m-3 \\
2
\end{array}\right) u^{m-1} \otimes x_{m-1}+u^{m-2} \otimes \mathrm{Sq}^{1} x_{m-1}, \\
u^{m-2} \otimes x_{m-2} & \mapsto u^{m-1} \otimes \mathrm{Sq}^{1} x_{m-2}+(m-1) u^{m-1} \otimes Q^{1} x_{m-2} \\
u^{m-1} \otimes x_{m-3} & \mapsto u^{m-1} \otimes w_{1}^{2} x_{m-3} .
\end{aligned}
$$

(Note: the second line is understood to mean that for $m$ even, $\mathrm{Sq}^{2}\left(u^{m-2} \otimes x_{m-1}\right)$ $=u^{m-1} \otimes w_{1} x_{m-1}$ )

Lemma 35. Let $x \in H^{r}(M)$ and $m-1 \geqslant a \geqslant m-r$. Then multiplication by $g^{*}(u)^{2}$ in $H^{*}\left(U^{*}\right)$ is given by $g^{*}(u)^{2} \cdot u^{a} \otimes x=u^{a+2} \otimes x$.

Proof. This is similar to Lemma 19 of $\S 5$ which describes multiplication by $g^{*}(u)$.

9. This section contains a description of the sets $\operatorname{Imm}\left(M^{m}, R^{2 m-i}\right)(i \leqslant 2)$ and $\operatorname{Emb}\left(M^{m}, R^{2 m}\right)$ for certain manifolds $M$. The results are a consequence of the filtration defined on the above sets in $\$ 3$, and the calculations of the integral (and twisted integral) cohomology of $M^{*}$ and $U^{*}$ made in $\$ 7$. The reader should note several things. First, the immersion results only use the 2-torsion of $M$; thus the presence of odd torsion (in $M$ ) might effect calculations involving the integral cohomology of $U^{*}$ (for example of

$$
\left.\operatorname{ker} A^{2 m-3}: H^{2 m-3}\left(U^{*}, Z\right) \rightarrow H^{2 m-1}\left(U^{*}, Z_{2}\right)\right) \text {. }
$$

Second, more is known about the set $\operatorname{Imm}\left(M, R^{2 m-1}\right)$ than I am able to obtain using this method [13,pp.406-407]. It should be pointed out that my results agree with those of [13], and that the results of [13] are obtained by using the 
fibration $O / O(n) \rightarrow B O(n) \rightarrow B O$. Finally, the material in this paper may be used to describe the sets $\operatorname{Emb}\left(M, R^{2 m-i}\right)$ for $i=1,2$ and certain manifolds $M$. The descriptions are, in general, quite complicated, and so only results for real projective space are given.

The material in this section is arranged as follows: first, all the results for immersions are listed, and then their proofs are discussed. Next, the set $\operatorname{Emb}\left(M, R^{2 m}\right)$ is described. Finally, the results for real projective space $P^{n}$ are given. Recall that $M$ is always a compact, connected, differentiable manifold of dimension $m,\left\{x_{j, i}\right\}_{i \in I_{j}}$ is a $Z_{2}$-basis for $H^{j}\left(M ; Z_{2}\right)$, and if $w_{1} \neq 0, \hat{x}_{m-1}$ is the (unique) basis element with $\mathrm{Sq}^{1} \hat{x}_{m-1}=w_{1} \hat{x}_{m-1} \neq 0$. Also, the reader should recall that $H^{m-i}\left(M ; Z w_{1}\right) \simeq H_{i}(M ; Z)$ by Poincaré duality.

THEOREM 36. Let $m \geqslant 2$, then

$$
\operatorname{Imm}\left(M, R^{2 m}\right)= \begin{cases}Z, & \text { m even, } \\ Z_{2}, & \text { m odd } .\end{cases}
$$

(See the remark after Theorem 43 later in this section.)

THEOREM 37. Let $m \geqslant 4$, then $\operatorname{Imm}\left(M, R^{2 m-1}\right)=G_{0} \supset G_{1}$. If $m$ is even, no additional hypotheses are required; if $m$ is odd, assume $H^{m-1}\left(M ; Z w_{1}\right)$ has no elements of order 4 (elements of $\infty$-order are allowed). Then the following table describes $G_{1}$ and $G_{0} / G_{1}$.

\begin{tabular}{|c|c|c|c|}
\hline & \multicolumn{2}{|c|}{ M-orientable $\left(w_{1}=0\right)$} & M-nonorientable $\left(w_{1} \neq 0\right)$ \\
\hline \multirow[t]{2}{*}{$m \equiv 0$ (4) } & $G_{0} / G_{1}$ & $u^{m-1} \otimes H^{m-1}\left(M ; Z_{2}\right)$ & $u^{m-1} \otimes H^{m-1}\left(M ; Z_{2}\right)$ \\
\hline & $G_{1}$ & 0 & 0 \\
\hline \multirow[t]{2}{*}{$m \equiv 1$} & $G_{0} / G_{1}$ & $\left\{\begin{array}{l}Z_{2}: u^{m-2} \otimes x_{m} \\
u^{m-1} \otimes H^{m-1}\left(M ; Z w_{1}\right)\end{array}\right.$ & $\left\{\begin{array}{l}Z_{2}: u^{m-2} \otimes x_{m}, \\
u^{m-1} \otimes H^{m-1}\left(M ; Z w_{1}\right)\end{array}\right.$ \\
\hline & $G_{1}$ & $Z_{2}: u^{m-1} \otimes x_{m}$ & 0 \\
\hline \multirow[t]{2}{*}{$m \equiv 2(4)$} & $G_{0} / G_{1}$ & $u^{m-1} \otimes H^{m-1}\left(M ; Z_{2}\right)$ & $u^{m-1} \otimes H^{m-1}\left(M ; Z_{2}\right)$ \\
\hline & $G_{1}$ & $Z_{2}: u^{m-1} \otimes x_{m}$ & $Z_{2}: u^{m-1} \otimes x_{m}$ \\
\hline \multirow[t]{2}{*}{$m \equiv 3(4)$} & $G_{0} / G_{1}$ & $\left\{\begin{array}{l}Z_{2}: u^{m-2} \otimes x_{m} \\
u^{m-1} \otimes H^{m-1}\left(M ; Z w_{1}\right)\end{array}\right.$ & $\left\{\begin{array}{l}Z_{2}: u^{m-2} \otimes x_{m}, \\
u^{m-1} \otimes H^{m-1}\left(M ; Z w_{1}\right)\end{array}\right.$ \\
\hline & $G_{1}$ & $Z_{2}: u^{m-1} \otimes x_{m}$ & 0 \\
\hline
\end{tabular}


REMARK 38. For Theorems 37 and 39 the reader should recall the descriptions of $G_{0} / G_{1}, G_{1}, F_{0} / F_{1}, F_{1} / F_{2}$, and $F_{2}$ obtained in $\S 3$. For example, Theorem 37 says (provided the hypotheses are satisfied) that if $M$ is orientable and $m \equiv 0$ (4), then $\operatorname{Imm}\left(M, R^{2 m-1}\right)$ is (up to extension which is treated later in this section) in 1-1 correspondence with $Z_{2} \oplus Z_{2} \oplus\left(H^{m-1}\left(M ; Z w_{1}\right)=\right.$ $\left.H^{m-1}(M ; Z)\right)$. The other cases have similar interpretations.

THEOREM 39. Let $m \geqslant 6$ and assume there is an immersion of $M$ in $R^{2 m-2}$; then there exist groups $F_{2}$ and $F_{1}$ with $F_{2} \subset F_{1} \subset F_{0}=\operatorname{Imm}\left(M, R^{2 m-2}\right)$. If $m \equiv 2,3$ (4) and $w_{1}=0$, the secondary operation $\Psi$ is not determined; in each of those cases it does have 0-indeterminacy. If $m$ is even, assume $H^{m-2}\left(M ; Z w_{1}\right)$ contains no elements of order 4 (elements of $\infty$ order are allowed). Also if $m \equiv 2$ (4) and $w_{1} \neq 0$, assume

$$
w_{1}^{2} \cdot\left\{y \mid 0 \neq y \in E_{2}^{m-3}\left(M, Q^{1}\right)\right\} \subset \mathrm{Sq}^{1} H^{m-2}\left(M ; Z_{2}\right) \oplus w_{1}^{2} Q^{1} H^{m-4}\left(M ; Z_{2}\right) .
$$

Then the following table describes $F_{2}, F_{1} / F_{2}$, and $F_{0} / F_{1}$. (Note that for $m \equiv 2,3$ and $w_{1}=0, F_{2}$ is either 0 or $Z_{2}$.)

REMARK 40. The remark after Theorem 37 applies to Theorem 39. Thus in the above table if $M$ is orientable and $m \equiv 1(4), \operatorname{Imm}\left(M, R^{2 m-2}\right)$ is (up to extension) in 1-1 correspondence with $H^{m-2}\left(M ; Z_{2}\right) \oplus Z_{2}$. The elements $\left\{u^{m-1} \otimes x_{m-2, i}+u^{m-2} \otimes Q^{1} x_{m-2, i}\right\}$ listed in this case are in the kernel of $A^{2 m-3}$ and "sit" in $H^{2 m-3}\left(U^{*}, Z\right)$. The form of the elements (i.e. the fact that $F_{0} / F_{1}$ is not just $\left.\left\{u^{m-1} \otimes x_{m-2, i}\right\}\right)$ is important in determining the first extension of the sets.

PRoofs. For Theorem 36, recall that $U^{*}$ has the homotopy type of a $2 m-1$ dimensional compact manifold. So $H^{2 m-1}\left(U^{*}, Z\right)$ is either $Z$ or $Z_{2}$. Theorem 27 shows that for $m$ odd $H^{2 m-1}\left(U^{*}, Z\right)=Z_{2}$ and for $m$ even $E_{2}^{2 m-1}\left(U^{*}\right)=Z_{2}$. Thus for $m$ even $H^{2 m-1}\left(U^{*}, Z\right) \neq Z_{2}$ and the desired result follows.

One case of Theorems 37 and 39 is worked out. The others are similar. First, consider $\operatorname{Imm}\left(M, R^{2 m-1}\right)=G_{0} \supset G_{1}$ with $m \equiv 2(4)$ and $w_{1} \neq 0$. The results of $\S 3$ show that $G_{1} \cong \operatorname{coker} A^{2 m-3}: H^{2 m-3}\left(U^{*} ; Z g\right) \rightarrow H^{2 m-1}\left(U^{*}, Z_{2}\right)$ (note $m$ is even, so $\left.A^{2 m-3}=\mathrm{Sq}^{2}+g^{*}(u)^{2}\right)$, and $G_{0} / G_{1} \cong H^{2 m-2}\left(U^{*}, Z g\right.$ ). The proof of Theorem $27-g$ may be used to show that $H^{2 m-2}\left(U^{*} ; Z g\right)_{2}$ consists of $Z_{2}$-generators which reduce to the elements $u^{m-1} \otimes x_{m-1, i} \in H^{2 m-2}\left(U^{*}, Z_{2}\right)$ and that the image of the reduction map $\rho_{*}: H^{2 m-3}\left(U^{*} ; Z g\right) \rightarrow H^{2 m-3}\left(U^{*} ; Z_{2}\right)$ consists of the elements $u^{m-3} \otimes x_{m}$ and $u^{m-1} \otimes x_{m-2, i}+u^{m-2} \otimes Q^{1} x_{m-2, i}$. The calculations of Corollary 34 and Lemma 35 show that $A^{2 m-3}\left(\operatorname{im} \rho_{*}\right)=0$, and thus $G_{1} \cong \operatorname{coker} A^{2 m-3} \cong Z_{2}$ with generator $u^{m-1} \otimes x_{m}$.

Next, consider $\operatorname{Imm}\left(M, R^{2 m-2}\right)=F_{0} \supset F_{1} \supset F_{2}$ for $m \equiv 2$ (4) and $w_{1} \neq 0$. 


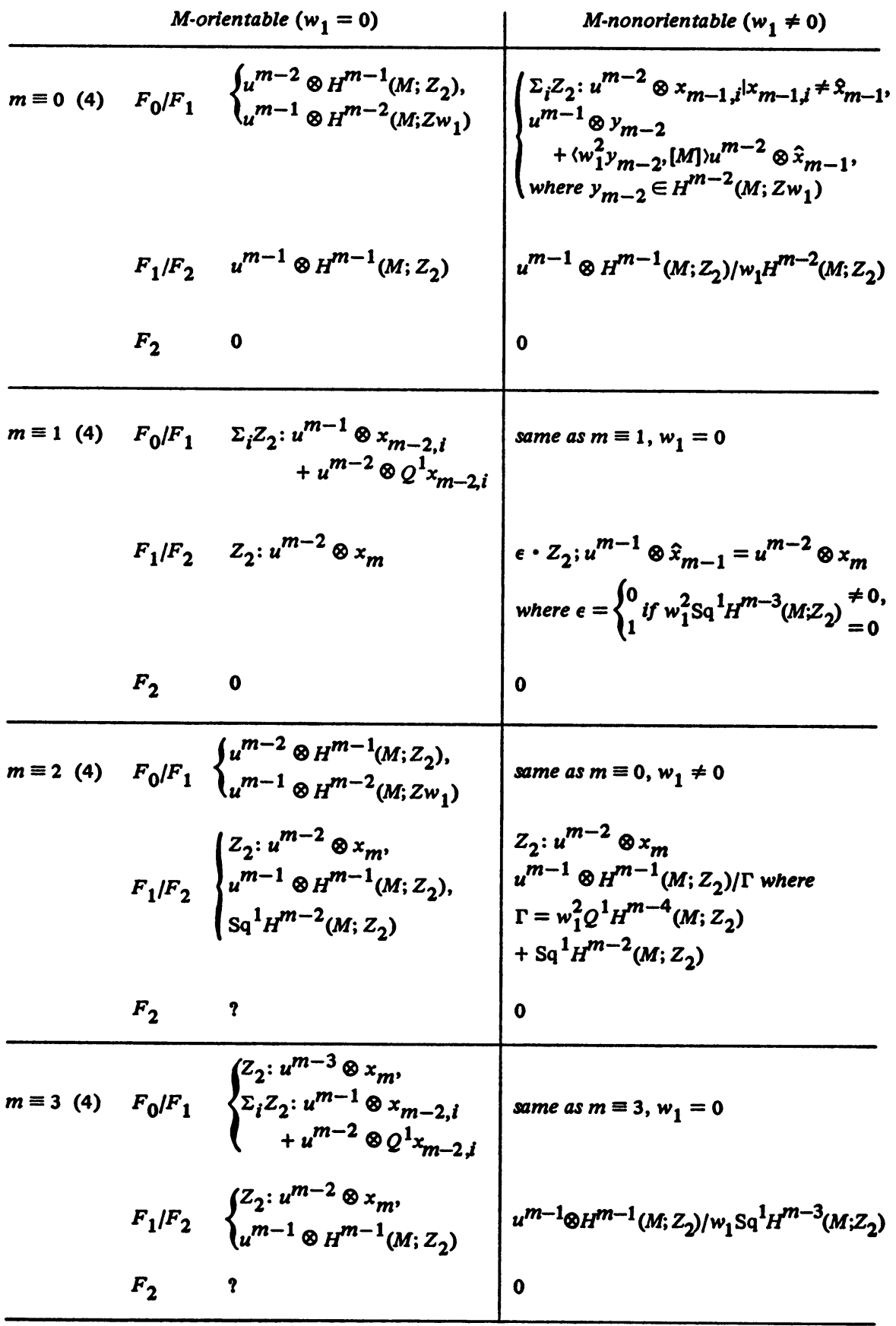

TABLE

The results of $\S 3$ show that $F_{0} / F_{1} \cong \operatorname{ker} A^{2 m-3}, F_{1} / F_{2} \cong \operatorname{coker} A^{2 m-4}$ and

$$
F_{2} \simeq \text { coker } \Psi \text { is } \begin{cases}\mathrm{Sq}^{2} H^{2 m-3}\left(U^{*} ; Z_{2}\right), & m \text { odd, } \\ \left(\mathrm{Sq}^{2}+g^{*}(u)^{2}\right) H^{2 m-3}\left(U^{*} ; Z_{2}\right), & m \text { even }\end{cases}
$$


The results of $\S 8$ show that for $m$ even that subset is $\left\{\left({ }^{m-1}\right) u^{m-1} \otimes x_{m}, u^{m-1}\right.$ $\otimes w_{1} x_{m-1, i}$, which is all of $H^{2 m-1}\left(U^{*}, Z_{2}\right)$ if either $w_{1} \neq 0$ or $w_{1}=0$ and $m \equiv 0$ (4). Thus $\Psi$ is onto (in the case being treated) and coker $\Psi=0$.

Theorem 27 may be used to show that

$$
\operatorname{im} \rho_{*}: H^{2 m-4}\left(U^{*} ; Z\right) \rightarrow H^{2 m-4}\left(U^{*} ; Z_{2}\right)
$$

is partially generated by the elements $u^{m-4} \otimes x_{m}, u^{m-1} \otimes Q^{1} x_{m-4, i}$, and $u^{m-2} \otimes x_{m-2, i}+u^{m-3} \otimes Q^{1} x_{m-2, i}$. Also, im $\rho_{*}$ might contain elements of the form $u^{m-1} \otimes y_{m-3}$ where $0 \neq y_{m-3} \in E_{2}^{m-3}\left(M, Q^{1}\right)$. Corollary 34 and Lemma 35 show that on the known elements in $\operatorname{im} \rho_{*}, A^{2 m-4}$ does the following:

$$
\begin{gathered}
u^{m-4} \otimes x_{m} \stackrel{A^{2 m-4}}{\longmapsto} 0, \\
u^{m-1} \otimes Q^{1} x_{m-4, i} \mapsto u^{m-1} \otimes w_{1}^{2} Q^{1} x_{m-4, i}, \\
u^{m-2} \otimes x_{m-2, i}+u^{m-3} \otimes Q^{1} x_{m-2, i} \mapsto u^{m-1} \otimes\left(w_{1} x_{m-2, i}+Q^{1} x_{m-2, i}\right) \\
=u^{m-1} \otimes \mathrm{Sq}^{1} x_{m-2, i}
\end{gathered}
$$

So $\operatorname{im} A^{2 m-4} \mid$ known elements $=\left\{u^{m-1} \otimes w_{1}^{2} Q^{1} x_{m-4, i}+\mathrm{Sq}^{1} x_{m-2, j}\right\}$. Since $A^{2 m-4}\left(u^{m-1} \otimes y_{m-3}\right)=u^{m-1} \otimes w_{1}^{2} y_{m-3}$, the assumption that

$$
w_{1}^{2} E_{2}^{m-3}\left(M, Q^{1}\right) \subset \mathrm{Sq}^{1} H^{m-2}\left(M ; Z_{2}\right) \oplus w_{1}^{2} Q^{1} H^{m-4}\left(M ; Z_{2}\right)
$$

insures that $E_{2}^{m-3}\left(M, Q^{1}\right)$ plays no role in coker $A^{2 m-4}$. Thus coker $A^{2 m-4}$ is isomorphic to the set of elements listed in the theorem.

Finally, Theorem 27 may also be used to show that $\operatorname{im} \rho_{*}: H^{2 m-3}\left(U^{*} ; Z\right)$ $\rightarrow H^{2 m-3}\left(U^{*} ; Z_{2}\right)$ consists of the elements $u^{m-2} \otimes x_{m-1, i} ; u^{m-1} \otimes Q^{1} x_{m-3, i}$; $u^{m-1} \otimes y_{m-2, i}$ where $y_{m-2, i}$ is the reduction of a $Z$-summand in $H^{m-2}\left(M ; Z w_{1}\right)$. The same lemmas used for $A^{2 m-4}$ above show that $A^{2 m-3}$ takes the following values on $\operatorname{im} \rho_{*}$ :

$$
\begin{aligned}
u^{m-2} \otimes x_{m-1, i} & \stackrel{A^{2 m-3}}{\longmapsto} u^{m-1} \otimes w_{1} x_{m-1, i}, \\
u^{m-1} \otimes Q^{1} x_{m-3, i} & \mapsto u^{m-1} \otimes w_{1}^{2} Q^{1} x_{m-3, i}, \\
u^{m-1} \otimes y_{m-2, i} & \mapsto u^{m-1} \otimes w_{1}^{2} y_{m-2, i}
\end{aligned}
$$

Thus $A^{2 m-3}$ is onto, and

$$
\operatorname{ker} A^{2 m-3} \simeq F_{0} / F_{1} \simeq\left\{\begin{aligned}
& u^{m-2} \otimes x_{m-1, i} \text { where } x_{m-1, i} \neq \hat{x}_{m-1}, \\
& u^{m-1} \otimes z_{m-2}+\left\langle w_{1}^{2} z_{m-2},[M]\right\rangle u^{m-2} \otimes x_{m-1} \\
& \text { where } z_{m-2} \in H^{m-2}\left(M ; Z w_{1}\right)
\end{aligned}\right.
$$

In order to deal with the extension problem in Theorems 37 and 39 recall that an exact sequence of abelian groups $0 \rightarrow V \stackrel{i}{\rightarrow} E \stackrel{j}{\rightarrow} W \rightarrow 0$ with $V$ a $Z_{2}$. vector space is completely determined by a map $B: W \cap$ \{elements of order 2 \} 
$\rightarrow V$. If $x \in W$ and $2 x=0$, then $B(x)=i^{-1} 2 j^{-1}(x)$. In particular the exact sequence

$$
0 \rightarrow \operatorname{coker} A^{2 m-4} \rightarrow\left\langle U^{*} \vee P^{2 m-3}, P(2)\right\rangle \rightarrow \operatorname{ker} A^{2 m-3} \rightarrow 0
$$

(from §3) is determined by a map $B^{2 m-4}: \operatorname{ker} A^{2 m-3} \cap$ \{elements of order 2 \} $\rightarrow \operatorname{coker} A^{2 m-4}$. An application of Theorem 4.1 of [13] shows that $B^{2 m-4}$ may be described as follows: let $\alpha \in H^{2 m-3}\left(U^{*} ; Z\right)$ with $A^{2 m-3}(\alpha)=0$ and $2 \alpha=0$, then

$$
B^{2 m-4}(\alpha)= \begin{cases}\mathrm{Sq}^{2} \delta^{-1} \alpha, & m \text { odd, } \\ \left(\mathrm{Sq}^{2}+g^{*}(u)^{2}\right) \delta^{-1} \alpha, & m \text { even, }\end{cases}
$$

where $\delta: H^{2 m-4}\left(U^{*} ; Z_{2}\right) \rightarrow H^{2 m-3}\left(U^{*} ; Z\right)$ is the coboundary (thus $B^{2 m-4}=$ $\left.A^{2 m-4} \delta^{-1}\right)$. The same description is also valid for $\operatorname{Emb}\left(M, R^{2 m-2}\right)$ but it is of more interest in the case of immersions since $F_{2}=0$ and thus $\left\langle U^{*} \vee P^{2 m-3}\right.$, $P(2)\rangle=\operatorname{Imm}\left(M, R^{2 m-1}\right)$ in six cases out of eight.

The description of $B$ is slightly more complicated for $\operatorname{Emb}\left(M, R^{2 m-1}\right)$ and $\operatorname{Imm}\left(M, R^{2 m-1}\right)$. For embeddings the same Theorem 4.1 shows that the sequence

$$
0 \rightarrow \operatorname{coker} A^{2 m-3} \rightarrow \operatorname{Emb}\left(M, R^{2 m-1}\right) \rightarrow H^{2 m-2}\left(M^{*} ; Z g\right) \rightarrow 0
$$

is determined by

$$
B^{2 m-3}=\left(A^{2 m-3}+g^{*}(u)^{2}+g^{*}(u) \mathrm{Sq}^{1}\right) \delta^{-1} .
$$

The same formula also holds for the set $\operatorname{Imm}\left(M, R^{2 m-1}\right)$.

The next two propositions describe the nontrivial extensions in Theorems 37 and 39.

Proposition 41. All the extensions in $\operatorname{Imm}\left(M, R^{2 m-1}\right)$ are trivial with the exception of the following two cases:

Case 1. $m \equiv 2$ (4), $w_{1} \neq 0: \operatorname{Imm}\left(M, R^{2 m-1}\right) \cong Z_{4} \oplus \operatorname{ker} \operatorname{Sq}^{1}: H^{m-1}\left(M ; Z_{2}\right)$ $\rightarrow H^{m}\left(M ; Z_{2}\right)$. (Note that $\mathrm{Sq}^{1}$ is onto in this case.)

Case 2. $m \equiv 3(4), w_{1}=0: \operatorname{Imm}\left(M, R^{2 m-1}\right) \cong Z_{4} \oplus H^{m-1}(M ; Z)$.

PROPOSITION 42. The first extension in $\operatorname{Imm}\left(M, R^{2 m-2}\right)$ has the following description.

Case 1. $m \equiv 0$ (4), $w_{1}=0$ : The extension is trivial.

$w_{1}=0:$ There is an exact sequence

$$
\begin{aligned}
0 & \rightarrow H^{m-1}\left(M ; Z_{2}\right) / w_{1} H^{m-3}\left(M ; Z_{2}\right) \rightarrow E \\
& \rightarrow\left\{H^{m-1}\left(M ; Z_{2}\right)-\hat{x}_{m-1}\right\} \oplus H^{m-2}\left(M ; Z w_{1}\right) \rightarrow 0
\end{aligned}
$$

with $B\left(x_{m-1, i}\right)=x_{m-1, i}$ and $B \mid H^{m-2}\left(M ; Z w_{1}\right)=0$.

Case 2. $m \equiv 1$ (4), $w_{1}=0$ : The extension is trivial. 
$w_{1} \neq 0:$ The extension is trivial if either $w_{1}^{2}=0$ or $w_{1}^{2} \operatorname{Sq}^{1} H^{m-3}\left(M ; Z_{2}\right)$ $\neq 0$. If $w_{1}^{2} \neq 0$ but $w_{1}^{2} \mathrm{Sq}^{1} H^{m-3}\left(M ; Z_{2}\right)=0$, then

$\operatorname{Imm}\left(M ; R^{2 m-2}\right) \cong Z_{4} \oplus \operatorname{ker} w_{1}^{2}: H^{m-2}\left(M ; Z_{2}\right) \rightarrow H^{m}\left(M ; Z_{2}\right)$.

Note that in the latter case $w_{1}^{2}$ is onto, so $\operatorname{dim} \operatorname{ker} w_{1}^{2}=\operatorname{dim} H^{m-2}\left(M, Z_{2}\right)-1$.

Case 3. $m \equiv 2(4), w_{1}=0$ : The extension is trivial. (Note: $F_{2}$ need not be zero.)

$w_{1} \neq 0:$ There is an exact sequence

$$
\begin{aligned}
0 & \rightarrow Z_{2} \oplus H^{m-1}\left(M ; Z_{2}\right) / \Gamma \rightarrow \operatorname{Imm}\left(M ; R^{2 m-2}\right) \\
& \rightarrow\left\{H^{m-1}\left(M ; Z_{2}\right)-\hat{x}_{m-1}\right\} \oplus H^{m-2}\left(M ; Z w_{1}\right) \rightarrow 0 .
\end{aligned}
$$

(See Theorem 39 for the definition of $\Gamma$.) Also $B \mid H^{m-1}\left(M, Z_{2}\right)=0$, and $B\left(Q^{1} x_{m-3}\right)=w_{1}^{2} x_{m-3}$.

Case 4. $m \equiv 3$ (4), $w_{1}=0$ : There is an exact sequence (note: $F_{2}$ need not be zero):

$$
\begin{aligned}
0 & \rightarrow Z_{2} \cdot u^{m-2} \otimes x_{m} \oplus H^{m-1}\left(M ; Z_{2}\right) \rightarrow\left\langle U^{*} \vee P^{2 m-3}, P(2)\right\rangle \\
& \rightarrow Z_{2} \cdot x_{m} \oplus H^{m-2}\left(M ; Z_{2}\right) \rightarrow 0
\end{aligned}
$$

and $B\left(x_{m}\right)=u^{m-2} \otimes x_{m}, B\left(x_{m-2}\right)=\mathrm{Sq}^{1} x_{m-2}$.

$w_{1} \neq 0$ : There is an exact sequence

$$
\begin{aligned}
0 & \rightarrow H^{m-1}\left(M ; Z_{2}\right) / w_{1} \operatorname{Sq}^{1} H^{m-3}\left(M ; Z_{2}\right) \rightarrow \operatorname{Imm}\left(M, R^{2 m-2}\right) \\
& \rightarrow Z_{2} \cdot x_{m} \oplus H^{m-2}\left(M ; Z_{2}\right) \rightarrow 0
\end{aligned}
$$

with $B\left(x_{m}\right)=0, B\left(x_{m-2}\right)=\mathrm{Sq}^{1} x_{m-2}$.

I would like to remark that the description of the first extension given here differs from the one I gave in [1]. After [1] was completed I discovered that in computing $\mathrm{Sq}^{1}\left(g^{*}(u)\right)$ in $H^{*}(P) \odot A_{2}\left(A_{2}\right.$ is the Steenrod algebra), I had forgotten the term $g^{*}(u) \otimes \mathrm{Sq}^{1}$; thus the description of the extensions in [1] is incorrect and must be modified by using the formulas given above.

The next result deals with $\operatorname{Emb}\left(M, R^{2 m}\right)$.

THEOREM 43.( $\left.{ }^{2}\right)$ If $m \geqslant 4$, then

$\operatorname{Emb}\left(M, R^{2 m}\right) \simeq H^{2 m-1}\left(M^{*} ; Z\right) \simeq \begin{cases}H^{m-1}(M ; Z), & \text { modd } w_{1}=0, \\ H^{m-1}(M ; Z), & \text { modd } w_{1} \neq 0, \\ H^{m-1}(M ; Z), & \text { m even } w_{1}=0, \\ Z \oplus \operatorname{ker~Sq}{ }^{1}: H^{m-1}\left(M ; Z_{2}\right) & \rightarrow H^{m}\left(M ; Z_{2}\right), \\ & m \text { even } w_{1} \neq 0 .\end{cases}$

( $\left.{ }^{2}\right)$ This description of $H^{2 m-1}\left(M^{*} ; Z\right)$ was obtained by Professor W. S. Massey by other methods and proved helpful to the present author in coming to some understanding of the integral cohomology of $M^{*}$. 
(Note: if $w_{1} \neq 0, \mathrm{Sq}^{1}: H^{m-1}\left(M ; Z_{2}\right) \rightarrow H^{m}\left(M ; Z_{2}\right)$ is onto, so dim $\mathrm{ker} \mathrm{Sq}^{1}=$ $\operatorname{dim} H^{m-1}\left(M ; Z_{2}\right)-1$.)

REMARK 44. Two comments are in order about Theorems 36 and 43. First, Hirsch [11] showed that for $m$ even, two immersions of $M$ in $R^{2 m}$ are regularly homotopic if and only if they had the same normal class (in $H^{m}\left(M ; Z w_{1}\right)$ $=Z)$. Moreover, he showed every even class in $H^{m}\left(M ; Z w_{1}\right)$ is the normal class of some immersion. Second, Malyi [14] showed that for $m$ even $(m>2), w_{1} \neq 0$, and any integer $\chi$ with $\chi \equiv 2 \sigma_{m}$ (4) $\left(\sigma_{m}=0\right.$ if $\bar{w}_{1} \bar{w}_{n-1}=0, \sigma_{m}=1$ if $\bar{w}_{1} \bar{w}_{n-1}$ $\neq 0)$, there exists an embedding $f: M \rightarrow R^{2 m}$ with normal number $\chi_{f}=\chi\left(\chi_{f}\right.$ $=\left\langle v_{f},[M]\right\rangle$ where $\nu_{f}$ is the normal class of $f$ and $[M]$ is the twisted fundamental class). Thus the presence of the $Z$ in both Theorems 1 and 4 is known and may be "detected" by geometric means.

Proof of THeOREM 43. The statements involving the 2-torsion of $H^{2 m-1}\left(M^{*} ; Z\right)$ are easy consequences of the general results about the Bockstein spectral sequence of $M^{*}$. An application of the Serre spectral sequence to the fibration $M \times M-\Delta \rightarrow M^{*} \rightarrow P^{\infty}$ shows that the odd torsion in $H^{*}\left(M^{*} ; Z\right)$ is just the group of elements of $H^{*}(M \times M-\Delta ; Z)$ invariant under the action induced by the twist map $T: M \times M \rightarrow M \times M$. In dimension $2 m-1$ that group is easily determined by studying the exact sequence of the pair $(M \times M, M \times M-\Delta)$ and using (when $M$ is orientable) the Thom Isomorphism

$$
H^{m-1}(M ; Z) \cong H^{2 m-1}(M \times M, M \times M-\Delta ; Z) .
$$

The following table describes the set of embeddings and immersions of real projective space $P^{m}$ in $R^{2 m-i}$ for $i \leqslant 2$. The results for immersions agree with those of Robinson [20] who studies the Postnikov factorization of $O / O(n) \rightarrow$ $B O(n) \rightarrow B O$.

\begin{tabular}{l|lll|ll}
\multicolumn{3}{c}{$\operatorname{Emb}\left(P^{n}, R^{2 n-i}\right)$} \\
\hline \multirow{1}{*}{$i=0$} & $Z$, & $n$ even, & $n \geqslant 4$ & $Z$, & \multicolumn{3}{c}{$\operatorname{Imm}\left(P^{n}, R^{2 n-i}\right)$} \\
& $Z_{2}$, & $n$ odd & & $Z_{2}$, & $n$ odd \\
$Z_{2}$, & $n$ even, & $n \geqslant 6$ & $Z_{2}$, & $n \equiv 0(4), n \geqslant 4$ \\
$Z_{2}$, & $n \equiv 1(4)$, & $n \neq 2^{r}$ & $Z_{2}+Z_{2}+Z_{2}$, & $n \equiv 1$, \\
$Z_{4}$, & $n \equiv 3(4)$, & & $Z_{4}$, & $n \equiv 2$, \\
& & & & $Z_{4}+Z_{2}$, & $n \equiv 3$, \\
& & $n \equiv 0(4)$, & $n \geqslant 8$ & 0, & $n \equiv 0(4), n \geqslant 6$ \\
$Z_{2}+Z_{2}+Z_{2}$, & $n \equiv 1$, & $n \neq 2^{r}$ & $Z_{2}+Z_{2}$, & $n \equiv 1$, & $n \neq 2^{r}$ \\
0, & $n \equiv 2$, & $2^{r}+1$ & $Z_{2}$, & $n \equiv 2$, \\
$Z_{4}+Z_{4} ? Z_{2}$, & $n \equiv 3$, & & $Z_{4}+Z_{4} ? Z_{2}$, & $n \equiv 3$,
\end{tabular}


10. This section deals with the existence of an embedding of $M^{m}$ in $R^{2 m-2}$. It is well known that every manifold $M^{m}$ embeds in $R^{2 m}$, and that if $m \geqslant 5, M^{m}$ embeds in $R^{2 m-1}$ if and only if $\bar{w}_{m-1}=0$ [10]. The following result is not best possible, but it does give the existence of embeddings of $M^{m}$ in $R^{2 m-2}$ in many cases. All cohomology groups in this section have $Z_{2}$ coefficients unless other coefficients are indicated.

THEOREM 45. Let $M$ be a manifold of dimension $m \geqslant 8$ with $\bar{w}_{m-i}=0$ for $i \leqslant 4$. If $\mathrm{Sq}^{2} H^{m-2}(M) \neq 0$, then there exists an embedding of $M$ in $R^{2 m-2}$. If $\mathrm{Sq}^{2} H^{m-2}(M)=0$, then there exists an embedding of $M$ in $R^{2 m-2}$ in the following cases:
(1) $w_{1}=0 ; m \equiv 0,1$
(4) always,
$m \equiv 2$
(4) if $\mathrm{Sq}^{1} H^{m-2}(M)=0$,
$m \equiv 3$
(4) if $\mathrm{Sq}^{3} H^{m-4}(M)=0$;
(2) $w_{1} \neq 0, m \equiv 0$
(4) if $w_{1} H^{m-2}(M) \subseteq w_{1}^{2} H^{m-3}(M)$,
$m \equiv 1$
(4) if $\left\{w_{1} \mathrm{Sq}^{1} H^{m-3}(M)+\left(\mathrm{Sq}^{1}+w_{1}\right) H^{m-2}(M)\right\}$$$
\subseteq\left\{\mathrm{Sq}^{2} H^{m-3}(M)+\mathrm{Sq}^{1} H^{m-2}(M)+w_{1}^{2} H^{m-3}(M)\right\},
$$

$m \equiv 2,3$ (4) always.

REMARK 46. A result of Massey's [15] shows that if $\alpha(m) \geqslant 5(\alpha(m)$ is the number of ones in the dyadic expansion of $m$ ) then $\bar{w}_{m-i}=0$ for $i \leqslant 4$.

Proof of TheOREM 45. Since $m \geqslant 8$, it suffices (in view of Haefliger's theorem) to produce a lift of $g: M^{*} \rightarrow P^{\infty}$ to $P^{2 m-3}$. The following Postnikov factorization of $p: P^{2 m-3} \rightarrow P^{\infty}$ is used to do this:

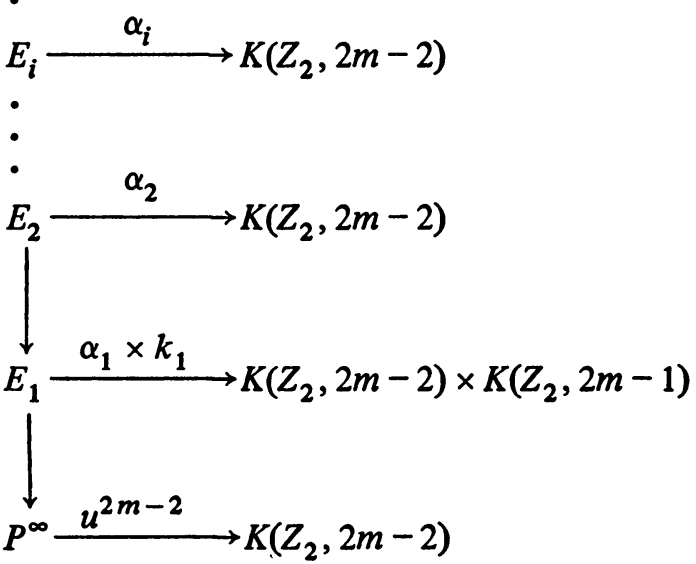

It differs from the one considered in $\S 3$ in that the $Z$ occuring in $\pi_{2 m-3}\left(S^{2 m-3}\right)$ is not killed all at once but rather "a $Z_{2}$ at a time". Thus if $g$ : $X \rightarrow P^{\infty}$ and $g^{*}\left(u^{2 m-2}\right)=0$, the $\alpha_{i}$-invariants correspond to looking at 
$B_{i}\left(g^{*}(u)^{2 m-3}\right)$ where $B_{i}$ is the $i$ th Bockstein. The lemma at the end of the proof shows that the assumption $\bar{w}_{m-i}=0$ for $i \leqslant 4$ implies that $0=g^{*}(u)^{2 m-4} \in$ $H^{*}\left(M^{*}\right)$ so the $\alpha_{i}(i>0)$ obstructions (for $M^{*}$ ) present no problem. The method of Thomas [12] is used to evaluate the $k_{1}$-obstruction.

So consider the commutative diagram:

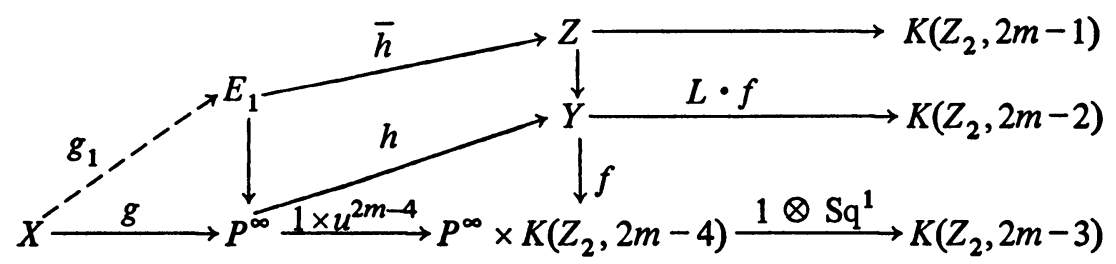

In the diagram $L$ is the following operation in $A\left(P^{\infty}\right)$ :

$$
L= \begin{cases}1 \otimes \mathrm{Sq}^{2}+u^{2} \otimes 1, & m \text { even, } \\ 1 \otimes \mathrm{Sq}^{2}, & m \text { odd }\end{cases}
$$

Also the space $Y(Z)$ is obtained by pulling back the path fibration via $1 \otimes$ $\mathrm{Sq}^{1}(L \cdot f)$. Clearly $\left(1 \otimes \mathrm{Sq}^{1}\right)\left(1 \times u^{2 m-4}\right)=0$, so a lift $h$ exists. Moreover, an easy calculation shows that $L \cdot f \cdot h=u^{2 m-2}$, so $\bar{h}$ exists making the square commute. Now, when the cohomology of $E_{1}$ and $Z$ is commuted (in dimension $\leqslant 2 m-1)$ it is seen that a class $k_{1}^{\prime} \in H^{2 m-1}(Z)$ may be chosen with two properties:

(1) $\bar{h}^{*}\left(k_{1}^{\prime}\right)$ is a representative for the first obstruction (i.e. for $\left.k_{1}\right)$.

(2) $k_{1}^{\prime}$ is a representative for the secondary operation $\Phi$ corresponding to the relation $L \cdot L+\left(\mathrm{Sq}^{1} L\right) \cdot \mathrm{Sq}^{1}=0$ in $A\left(P^{\infty}\right)$.

If $g^{*}(u)^{2 m-2}=0$, there exists a lift $g_{1}$ and it is easily checked (using the above calculation of $H^{2 m-1}\left(E_{1}\right)$ ) that the obstruction set $k_{1}(g)=\bigcup_{g_{1}} g_{1}^{*}\left(k_{1}\right)$ is a coset of $L \cdot H^{2 m-3}(X)$. The same condition implies that $\Phi\left(g^{*}\left(u^{2 m-4}\right)\right)$ is defined and is a coset of $L H^{2 m-3}(X)+\mathrm{Sq}^{1} L H^{2 m * 4}(X)$. Properties (1) and (2) of the class $k_{1}^{\prime}$ show that when

$$
\mathrm{Sq}^{1} L H^{2 m-4}(X) \subset L H^{2 m-3}(X)
$$

the two sets $k_{1}(g)$ and $\Phi\left(g^{*}\left(u^{2 m-4}\right)\right)$ coincide.

For the space $M^{*}$ easy calculations show that $(* *)$ occurs precisely under the conditions of the theorem. Finally, the assumption $\bar{w}_{m-i}=0$ for $i \leqslant 4$ and the following lemma show that $g^{*}(u)^{2 m-4}=0$. Thus $0 \in \Phi\left(g^{*}(u)^{2 m-4}\right)$ and so a lift of $g$ to $E_{2}$ and hence to $P^{2 m-3}$ exists.

LEMma 47. Let $k \leqslant m$. Then

$$
g^{*}(u)^{2 m-k}=\left(u \otimes 1^{2}\right)^{2 m-k}=\sum_{i=k}^{[2 m-k-1 / 2]} u^{2 m-k-2 i} \otimes \bar{w}_{i}^{2}
$$

in $H^{2 m-k}\left(M^{*}\right)$. 
ProOF.

$$
\begin{aligned}
\left(u \otimes 1^{2}\right)^{2 m-k} & =\xi\left(u^{2 m-k} \otimes 1^{2}\right)=\sum_{i=0}^{[2 m-k-1 / 2]} u^{2 m-k-2 i} \otimes\left(\bar{Q}^{i}(1)\right)^{2} \\
& =\sum_{i=k}^{[2 m-k-1 / 2]} u^{2 m-k-2 i} \otimes \bar{w}_{i}^{2}
\end{aligned}
$$

since $\bar{Q}^{i}(1)=\bar{w}_{i}$ and $\sum_{i=0}^{k-1} u^{2 m-k-2 i} \otimes d H^{i}(M)=0$ in $H^{2 m-k}\left(M^{*}\right)$ by the result of Yo.

\section{BIBLIOGRAPHY}

1. D. Bausum, Embeddings and immersions of manifolds in Euclidean space, Ph. D. Thesis, Yale University, New Haven, Conn., 1974.

2. S. Eilenberg, Homology of spaces with operators. I, Trans. Amer. Math. Soc. 61 (1947), 378-417; errata, ibid. 62 (1947), 548. MR 9, 52.

3. S. Feder, The reduced symmetric product of projective spaces and the generalized Whitney theorem, Illinois J. Math. 16 (1972), 323-329. MR 45 \#6025.

4. S. Gitler, Immersion and embedding of manifolds, Proc. Sympos. Pure Math., vol. 22, Amer. Math. Soc., Providence, R. I., 1971, pp. 87-96. MR 47 \#4275.

5. R. Greenblatt, The twisted Bockstein coboundary, Proc. Cambridge Philos. Soc. 61 (1965), 295-297. MR 30 \#5314.

6. D. Handel, An embedding theorem for real projective spaces, Topology 7 (1968), 125-130. MR 37 \#3582.

7. A. Haefliger, Points multiples d'une application et produit cyclique réduit, Amer. J. Math. 83 (1961), 57-70. MR.22 \#11404.

8. - Plongements différentiables dans le domaine stable, Comment. Math. Helv. 37 (1962/63), 155-176. MR 28 \#625.

9. A. Haefliger and M. W. Hirsch, Immersions in the stable range, Ann. of Math. (2) 75 (1962), 231-241. MR 26 \#784.

10. On the existence and classification of differentiable embeddings, Topology 2 (1963), 129-135. MR 26 \#6981.

11. M. W. Hirsch, Immersions of manifolds, Trans. Amer. Math. Soc. 93 (1959), 242276. MR 22 \#9980.

12. I. M. James and E. Thomas, Note on the classification of cross-sections, Topology 4 (1965), 351-359. MR 35 \#3685.

13. L. L. Larmore and E. Thomas, Groups extensions and twisted cohomology theories, Illinois J. Math. 17 (1973), 397-410.

14. B. D. Malyĩ, On the Whitney-Mahowald theorem concerning the normal numbers of smooth embeddings, Mat. Zametki 5 (1969), 91-97 = Math. Notes 5 (1969), 57-60. MR 40 \#3569.

15. W. S. Massey, On the Steifel-Whitney classes of a manifold, Amer. J. Math. 82 (1960), 92-102. MR 22 \#1918.

16. W. S. Massey and F. P. Peterson, The cohomology structure of certain fiber spaces. I, Topology 4 (1965), 47-65. MR 32 \#6459.

17. J. F. McClendon, Higher order twisted cohomology operations, Invent. Math. 7 (1969), 183-214. MR 40 \#3541. \#6002.

18. - Obstruction theory in fiber spaces, Math. Z. 120 (1971), 1-17. MR 45

19. C. A. Robinson, Moore-Postnikov systems for non-simple fibrations, Illinois J. Math. 16 (1972), 234-242. MR 45 \#7714.

20. - Stable homotopy theory over a fixed base space, Bull. Amer. Math. Soc. 80 (1974), 248-252.

21. Emery Thomas, Seminar on fiber spaces, Lecture Notes in Math., vol. 13, SpringerVerlag, Berlin and New York, 1966. MR 34 \#3582.

22. - Postnikov invariants and higher order cohomology operations, Ann. of Math. (2) 85 (1967), 184-217. MR 35 \#1029. 
23. T. Yasui, Corrections to "The reduced symmetric product of a complex projective space and the embedding problem", Hiroshima Math. J. 2 (1972), 233-237.

24. - Note on the embeddings of real projective spaces, Hiroshima Math. J. 3 (1973), 409-418..

25. G. Yo (Yueh Ching-chung), Cohomology operations and duality in a manifold, Sci. Sinica 12 (1963), 1469-1487. MR 31 \#6227.

26. Coholomogy mod $p$ of deleted cyclic product of a manifold, Sci. Sinica 12 (1963), 1779-1794. MR 31 \#6228.

27. Cohomology of the projective bundle of a manifold and its application to immersions, Sci. Sinica 14 (1965), 959-963. MR 32 \#1723.

DEPARTMENT OF MATHEMATICS, YALE UNIVERSITY, NEW HAVEN, CONNECT. ICUT 06511

Current address: School of Mathematics, University of Minnesota, Minneapolis, Minnesota 55455 